\title{
Super acid catalysed sequential hydrolysis/cycloisomerization of $o$-(acetylenic)benzamides under microwave condition: Synthesis, antinociceptive and antiinflammatory activity of substituted isocoumarins
}

\author{
CHANDRASEKARAN PRAVEEN ${ }^{\mathrm{a}}, \mathrm{P}^{\mathrm{D}}$ DHEENKUMAR ${ }^{\mathrm{b}}$ and P T PERUMAL ${ }^{\mathrm{a}, *}$ \\ ${ }^{a}$ Organic Chemistry Division, Central Leather Research Institute (CSIR), Adyar, Chennai 600 020, India \\ ${ }^{b}$ Department of Pharmaceutical Chemistry, EGS Pillay College of Pharmacy, Nagapattinam 611 002, India \\ e-mail: ptperumal@gmail.com
}

MS received 28 November 2011; revised 12 May 2012; accepted 10 July 2012

\begin{abstract}
Synthesis of isocoumarins and related compounds via triflic acid promoted hydrolysis/cyclization sequence of 2-(alkynyl)benzamides under microwave condition was achieved. The substrate scope of the reaction was broad to include not only aromatic but also polyaromatic and heteroaromatic motifs, thus highlighting the significance of this methodology. One-pot operation, short reaction time, good chemical yields and excellent regioselectivity are the advantages of this protocol. All the synthesized compounds were evaluated for their antinociceptive and antiinflammatory activities using in vivo rodent models.
\end{abstract}

Keywords. Microwave; sequential process; isocoumarins; antinociceptive; antiinflammatory.

\section{Introduction}

Trifluoromethane sulphonic acid $\left(\mathrm{CF}_{3} \mathrm{SO}_{3} \mathrm{H}\right.$ or HOTf $)$, also known as triflic acid, is the strongest Brø̈nsted acid (pKa -13.6) available at industrial scale. ${ }^{1}$ It is one of the small group of acids commonly known as 'super acids', being stronger than concentrated $\mathrm{H}_{2} \mathrm{SO}_{4}$. Amongst this special class of compounds, triflic acid has several important advantages. It is non-oxidizing, has a high thermal stability, is resistant to both oxidation and reduction and does not yield fluoride ions, even in the presence of strong nucleophiles. ${ }^{2}$ Unlike $\mathrm{H}_{2} \mathrm{SO}_{4}, \mathrm{FSO}_{3} \mathrm{H}$ or $\mathrm{ClSO}_{3} \mathrm{H}$, it does not lead to sulphonations and can be used in various protonation reactions. ${ }^{3}$ This novel collection of properties has made triflic acid an important reagent and catalyst in modern synthetic chemistry. As a comparatively new substance, it was first reported in $1954^{4}$ and subsequently commercialized, ${ }^{5}$ researchers continue to find new and interesting applications of triflic acid. ${ }^{6}$

On the other hand, development of effective strategies for the synthesis of a large structural diversity of heterocyclic compounds is a very important challenge in modern organic synthesis. ${ }^{7}$ In particular, the synthesis of low molecular weight heterocyclic compounds

*For correspondence with potential pharmacological properties has attracted considerable attention due to the need in identifying active compounds by high-throughput screening of large combinatorial libraries. ${ }^{8}$ Recently, a seminal study was carried out by Uchiyama et al. using HOTf as catalyst in the regioselective cyclization of 2-(alkynyl)benzoic acids leading to isocoumarins. ${ }^{9}$ Moreover, Bianchi et al. reported the direct synthesis of 3-substituted isocoumarins through silver catalysed heteroannulation of $o$-(1-alkynyl)benzamides. ${ }^{10}$ However, these methodologies are applicable for few substrates and a great deal of interest exists in the synthesis of isocoumarin library. ${ }^{11}$ In connection with our ongoing interest in developing new synthetic strategies for the construction of heterocycles $^{12}$ under HOTf acid catalysis, ${ }^{12 \mathrm{c}}$ we were interested in the sequential hydrolysis/cycloisomerization of 2(alkynyl)benzamides ${ }^{13}$ leading to isocoumarins, ${ }^{14}$ with particular emphasis on performing this transformation under microwave condition. ${ }^{15}$

\section{Experimental}

\subsection{Materials, methods and instruments}

All commercially available solvents and reagents were used without further purification. Anhydrous solvents were purchased from Sigma-Aldrich. All reactions of 
air and water sensitive materials were performed in flame dried glassware under a positive atmosphere of nitrogen or argon using standard syringe, cannula and septa techniques. Solutions in organic solvents were dried with anhydrous sodium sulphate. Solvents were evaporated under reduced pressure. Melting points were obtained using a Kofler-Galen hot stage microscope and are uncorrected. Infrared (IR) spectra were recorded on a Perkin-Elmer FTIR spectrophotometer as $\mathrm{KBr}$ pellets for solid compounds and neat sample for liquid compounds. ${ }^{1} \mathrm{H}$ and ${ }^{13} \mathrm{C}$ NMR spectra were obtained in $\mathrm{CDCl}_{3}$ and DMSO- $d_{6}$ on a JEOL spectrometer at 500 and $125 \mathrm{MHz}$, respectively. Proton chemical shifts $(\delta)$ are relative to tetramethylsilane (TMS, $\delta=0.00)$ as internal standard and expressed in parts per million. The number of protons $(n)$ for a given resonance was indicated as $n \mathrm{H}$. Spin multiplicities are given as s (singlet), $d$ (doublet), $t$ (triplet), dd (doublet of doublet), dt (doublet of triplet), ddd (doublet of doublet of doublet) and m (multiplet). Coupling constants $(J)$ are given in hertz. Mass spectra were recorded on a Thermo Finnigan LCQ Advantage MAX 6000 ESI mass spectrometer using electrospray ionization method. GC-MS were recorded using a Perkin-Elmer Auto System XL Gas Chromatograph with a TurboMass Mass Spectrometer (EI, $70 \mathrm{ev}$ ), with helium as carrier gas at a flow rate of $1 \mathrm{~mL} / \mathrm{min}$, Perkin-Elmer Elite series PE-5, capillary column $(30 \mathrm{~m} \times 0.25 \mathrm{~mm} \times$ $1 \mu \mathrm{m}$ ), oven programmed between 100 and $260^{\circ} \mathrm{C}$ at the rate of $10^{\circ} \mathrm{C} / \mathrm{min}$. Elemental analyses were recorded using a Thermo Finnigan FLASH EA $1112 \mathrm{CHN}$ analyzer. All the compounds gave $\mathrm{C}, \mathrm{H}$ and $\mathrm{N}$ analysis within $\pm 0.5 \%$ of the theoretical values. All microwave experiments were performed using a BPL microwave cooking system model BMO-700T, manufactured by BPL-SANYO Utilities and Appliances Ltd, Bangalore,

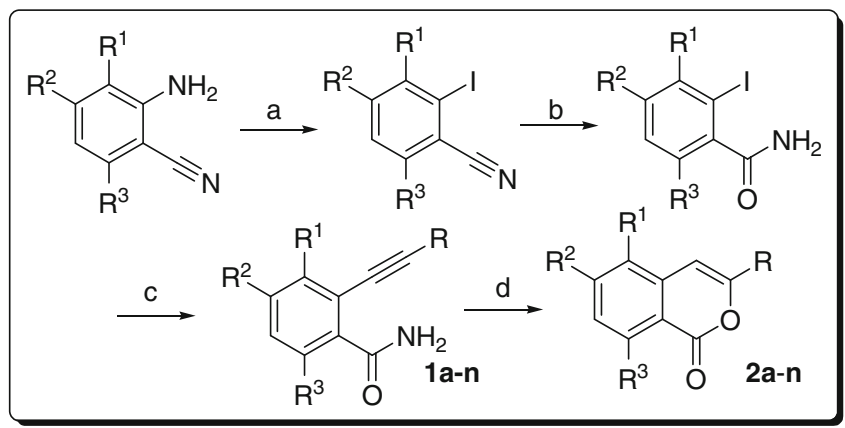

Scheme 1. Reagents and condition: (a) 1.1 equiv. $\mathrm{NaNO}_{2} / 25 \% \mathrm{HCl}, 0$ to $5^{\circ} \mathrm{C}, 30 \mathrm{~min}$, then 1.2 equiv. KI (aqueous), $5^{\circ} \mathrm{C}$ to rt, $4 \mathrm{~h}$; (b) $30 \% \mathrm{H}_{2} \mathrm{O}_{2} / 3.0$ equiv. $\mathrm{Na}_{2} \mathrm{CO}_{3}$, Acetone, $5^{\circ} \mathrm{C}$ to rt, $1 \mathrm{~h}$; (c) $5 \mathrm{~mol} \% \mathrm{Pd}\left(\mathrm{PPh}_{3} \mathrm{P}\right)_{2} \mathrm{Cl}_{2} / 5 \mathrm{~mol} \%$ $\mathrm{CuI} / \mathrm{Et}_{3} \mathrm{~N}, \mathrm{rt}, 15 \mathrm{~min}, \mathrm{~N}_{2}$ then 1.1 equiv. terminal alkyne; (d) 1.0 equiv. TfOH, toluene, $300 \mathrm{~W}, 3 \mathrm{~min}$.

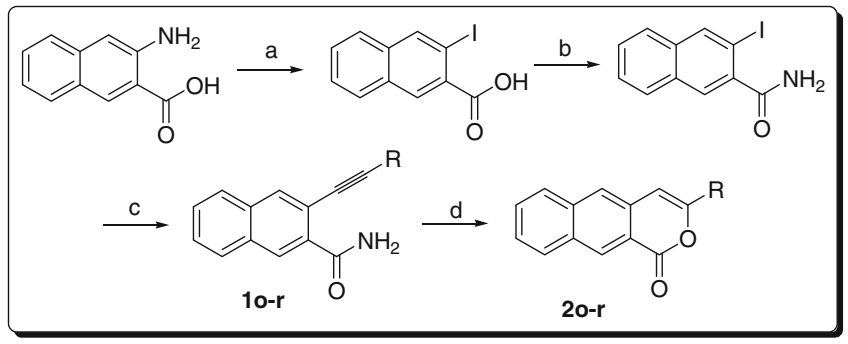

Scheme 2. Reagents and condition: (a) 1.1 equiv. $\mathrm{NaNO}_{2 / 2} \% \mathrm{HCl}, 0$ to $5^{\circ} \mathrm{C}, 30 \mathrm{~min}$, then 1.2 equiv. KI (aqueous), $5^{\circ} \mathrm{C}$ to $\mathrm{rt}, 4 \mathrm{~h}$; (b) 3.0 equiv. $\mathrm{PCl}_{5}, \mathrm{Et}_{2} \mathrm{O}, 0$ to $5^{\circ} \mathrm{C}, 1 \mathrm{~h}$ then excess $\mathrm{NH}_{3}$ (aq.), $0^{\circ} \mathrm{C}$ to $\mathrm{rt}, 2 \mathrm{~h}$; (c) $5 \mathrm{~mol} \%$ $\mathrm{Pd}\left(\mathrm{PPh}_{3} \mathrm{P}\right)_{2} \mathrm{Cl}_{2} / 5 \mathrm{~mol} \% \mathrm{CuI} / \mathrm{Et}_{3} \mathrm{~N}, \mathrm{rt}, 15 \mathrm{~min}, \mathrm{~N}_{2}$ then 1.1 equiv. terminal alkyne; (d) 1.0 equiv. $\mathrm{TfOH}$, toluene, $300 \mathrm{~W}$, $3 \mathrm{~min}$.

India. The oven has variable timing cycles from $1 \mathrm{~s}$ to $99 \mathrm{~min}$ and $99 \mathrm{~s}$ and heating cycles from 155 to $700 \mathrm{~W}$ of power output which can be varied by selecting power levels ranging from 10 to 100 . The overall dimension of the domestic oven is $525(\mathrm{~W}) \times 419$ (D) $\times 281(\mathrm{H})$ $\mathrm{mm}$ with a chamber of $350(\mathrm{~W}) \times 370(\mathrm{D}) \times 208(\mathrm{H})$ $\mathrm{mm}$. The microwave frequency is $50 \mathrm{~Hz}$ and the oven capacity is 26 litres. Column chromatography was performed using a mixture of petroleum ether and ethyl acetate on silica gel (100-200 mesh, SRL, India). Analytical TLC was performed on pre-coated plastic sheets of silica gel G/UV-254 of $0.2 \mathrm{~mm}$ thickness (MachereyNagel, Germany) using analytical grade solvents and visualizing with iodine spray $\left(10 \% \mathrm{w} / \mathrm{w}_{2}\right.$ in silica gel) or UV light ( $\lambda=254$ and $365 \mathrm{~nm})$. The inflammation was quantitated using a digital plethysmometer (Ugo Basile Company, Italy). The following reactions (schemes 1 to 3 ) were performed by published procedures: Sandmeyer iodination ${ }^{16}$ of 2-aminobenzonitriles and 3-aminonaphthalene-2-carboxylic acid, Alkaline hydrolysis of 2-iodobenzonitriles, ${ }^{17}$ Sonogashira crosscoupling of 2-halobenzamides, ${ }^{18}$ conversion of acid to

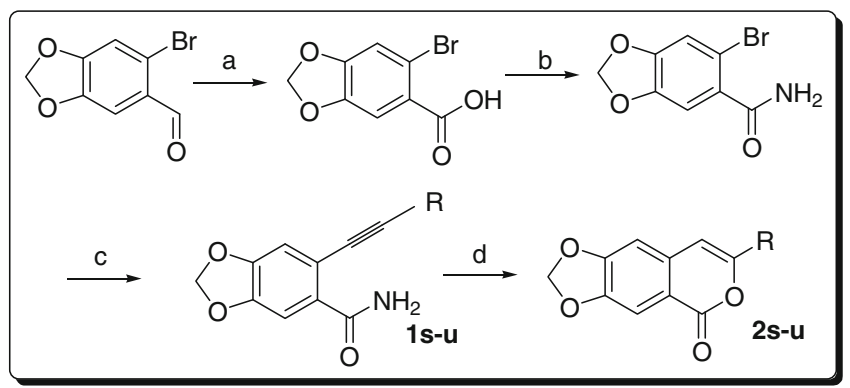

Scheme 3. Reagents and condition: (a) 1.0 equiv. oxone, DMF, rt, $1 \mathrm{~h}$; (b) 3.0 equiv. $\mathrm{SOCl}_{2}, \mathrm{Et}_{2} \mathrm{O}, 0$ to $5^{\circ} \mathrm{C}, 1 \mathrm{~h}$ then excess $\mathrm{NH}_{3}$ (aq.), $0^{\circ} \mathrm{C}$ to rt, $2 \mathrm{~h}$; (c) $5 \mathrm{~mol} \% \mathrm{Pd}\left(\mathrm{PPh}_{3} \mathrm{P}\right)_{2} \mathrm{Cl}_{2} /$ $5 \mathrm{~mol} \% \mathrm{CuI} / \mathrm{Et}_{3} \mathrm{~N}, \mathrm{rt}, 15 \mathrm{~min}, \mathrm{~N}_{2}$ then 1.1 equiv. terminal alkyne; (d) 1.0 equiv. TfOH, toluene, $300 \mathrm{~W}, 3 \mathrm{~min}$. 
amide, ${ }^{19}$ oxone promoted oxidation of 6-bromopiperanal, ${ }^{20}$ conversion of indole-2-carboxylic acid to indole-2-carbonitrile, ${ }^{21} \mathrm{C} 3$-iodination of indole, ${ }^{22}$ and $\mathrm{N}$-alkylation of indole derivatives. ${ }^{23}$

\subsection{General procedure for the synthesis of isocoumarin derivatives $\mathbf{2 a - 2 x}$}

Triflic acid $(1.0 \mathrm{mmoL})$ was added to a mixture of 2(alkynyl)benzamide $(1.0 \mathrm{mmoL})$ and toluene $(1 \mathrm{~mL})$ in a glass vial and inserted in an alumina bath $(100 \mathrm{~g}$, $60 \mathrm{G}_{254}$, Fischer scientific bath $(6.8 \mathrm{~cm}$ diameter $\left.)\right)$ and irradiated in a BPL-SANYO domestic microwave oven operating at $300 \mathrm{~W}$ at a pulse rate of $30 \mathrm{sec}$ for $3 \mathrm{~min}$. After cooling to room temperature, the reaction mixture was quenched with $10 \% \mathrm{NaHCO}_{3}$ solution, extracted with $\mathrm{CH}_{2} \mathrm{Cl}_{2}(3 \times 15 \mathrm{~mL})$ and the organic extract was dried with anhydrous $\mathrm{MgSO}_{4}$. Removal of the solvent under reduced pressure followed by purification with column chromatography afforded the pure product.

2.2a 3-Phenyl-1H-isochromen-1-one (2a): Brown solid; mp $85-87^{\circ} \mathrm{C}$ (lit. ${ }^{24} \mathrm{mp} 87-88^{\circ} \mathrm{C}$ ); IR (KBr): $1738,1665 \mathrm{~cm}^{-1} .{ }^{1} \mathrm{H}$ NMR $\left(500 \mathrm{MHz}, \mathrm{CDCl}_{3}\right): \delta_{\mathrm{H}} 6.95$ (s, 1H), 7.40-7.51 (m, 5H), $7.72(\mathrm{t}, 1 \mathrm{H}, J=7.3 \mathrm{~Hz})$, $7.88\left(\mathrm{dt}, 2 \mathrm{H}, J_{1}=1.5, J_{2}=8.1 \mathrm{~Hz}\right), 8.31(\mathrm{~d}, 1 \mathrm{H}$, $J=8.0 \mathrm{~Hz}) .{ }^{13} \mathrm{C} \mathrm{NMR}\left(125 \mathrm{MHz}, \mathrm{CDCl}_{3}\right): \delta_{\mathrm{C}} 101.8$, $120.5,125.2,126.0,128.1,128.8,129.7,130.0,132.0$, 134.8, 137.5, 153.6, 162.3. MS (EI): $m / z=222\left[\mathrm{M}^{+}\right]$. Anal. Calcd for $\mathrm{C}_{15} \mathrm{H}_{10} \mathrm{O}_{2}: \mathrm{C}, 81.07 ; \mathrm{H}, 4.54 \%$. Found: C, $80.95 ; \mathrm{H}, 4.52 \%$.

2.2b 3-(3-Fluorophenyl)-1H-isochromen-1-one (2b): Colourless solid; $\mathrm{mp} 136-138^{\circ} \mathrm{C}$ (lit. $\left.{ }^{25} \mathrm{mp} 138-140^{\circ} \mathrm{C}\right)$; IR (KBr): $1715 \mathrm{~cm}^{-1} \cdot{ }^{1} \mathrm{H}$ NMR $\left(500 \mathrm{MHz}, \mathrm{CDCl}_{3}\right)$ : $\delta_{\mathrm{H}} 6.94(\mathrm{~s}, 1 \mathrm{H}), 7.13\left(\mathrm{td}, 1 \mathrm{H}, J_{1}=8.0, J_{2}=\right.$ $2.5 \mathrm{~Hz}), 7.41-7.44(\mathrm{~m}, 1 \mathrm{H}), 7.50-7.59(\mathrm{~m}, 3 \mathrm{H}), 7.62-$ $7.77(\mathrm{~m}, 2 \mathrm{H}), 8.35(\mathrm{~d}, 1 \mathrm{H}, J=8.1 \mathrm{~Hz}) .{ }^{13} \mathrm{C}$ NMR $\left(125 \mathrm{MHz}, \mathrm{CDCl}_{3}\right): \delta_{\mathrm{C}} 102.5,112.1\left(J_{\mathrm{C}-\mathrm{F}}=24.0 \mathrm{~Hz}\right)$, $117.0\left(J_{\mathrm{C}-\mathrm{F}}=22.0 \mathrm{~Hz}\right), 120.6,120.8\left(J_{\mathrm{C}-\mathrm{F}}=3.0 \mathrm{~Hz}\right)$, $126.0,128.5,129.6,130.5\left(J_{\mathrm{C}-\mathrm{F}}=9.0 \mathrm{~Hz}\right), 134.0$ $\left(J_{\mathrm{C}-\mathrm{F}}=8 \mathrm{~Hz}\right), 135.0,137.1,152.2,161.9,163.1\left(J_{\mathrm{C}-\mathrm{F}}=\right.$ $245.0 \mathrm{~Hz})$. MS (EI): $m / z=240\left[\mathrm{M}^{+}\right]$. Anal. Calcd for $\mathrm{C}_{15} \mathrm{H}_{9} \mathrm{O}_{2} \mathrm{~F}: \mathrm{C}, 75.00 ; \mathrm{H}, 3.78 \%$. Found: C, 74.89; H, $3.82 \%$.

2.2c 3-(4-Methoxyphenyl)-1H-isochromen-1-one (2c): Colourless solid; mp $143-145^{\circ} \mathrm{C}$ (lit. ${ }^{26} \mathrm{mp} 142-143^{\circ} \mathrm{C}$ ); IR (KBr): $1727,1566,1478 \mathrm{~cm}^{-1} .{ }^{1} \mathrm{H}$ NMR $(500 \mathrm{MHz}$, $\left.\mathrm{CDCl}_{3}\right): \delta_{\mathrm{H}} 3.87(\mathrm{~s}, 3 \mathrm{H}) ; 6.80(\mathrm{~s}, 1 \mathrm{H}) ; 6.97(\mathrm{dt}, 2 \mathrm{H}$, $\left.J_{1}=9.2, J_{2}=2.8 \mathrm{~Hz}\right) ; 7.42-7.46(\mathrm{~m}, 2 \mathrm{H}) ; 7.70(\mathrm{td}$, $\left.1 \mathrm{H}, J_{1}=7.1, J_{2}=1.3 \mathrm{~Hz}\right) ; 7.83\left(\mathrm{dd}, 2 \mathrm{H}, J_{1}=9.2\right.$, $\left.J_{2}=2.1 \mathrm{~Hz}\right) ; 8.30(\mathrm{~d}, 1 \mathrm{H}, J=8.0 \mathrm{~Hz}) \cdot{ }^{13} \mathrm{C} \mathrm{NMR}$ $\left(125 \mathrm{MHz}, \mathrm{CDCl}_{3}\right): \delta_{\mathrm{C}} 55.5,100.1,114.1,114.2,120.0$,
124.6, 125.7, 126.7, 126.8, 127.6, 129.5, 134.9, 138.0, 153.7, 161.0, 162.4 162.5. MS (EI): $m / z=252\left[\mathrm{M}^{+}\right]$. Anal. Calcd for $\mathrm{C}_{16} \mathrm{H}_{12} \mathrm{O}_{3}: \mathrm{C}, 76.18 ; \mathrm{H}, 4.79 \%$. Found: C, $76.02 ; \mathrm{H}, 4.82 \%$.

2.2d 3-Butyl-1H-isochromen-1-one (2d): Brown solid; $\mathrm{mp} 45-47^{\circ} \mathrm{C}$ (lit. ${ }^{27} \mathrm{mp} 49.5-50.5^{\circ} \mathrm{C}$ ); IR (neat): 2958, 1731, $1657 \mathrm{~cm}^{-1} .{ }^{1} \mathrm{H}$ NMR $\left(500 \mathrm{MHz}, \mathrm{CDCl}_{3}\right)$ : $\delta_{\mathrm{H}} 0.94(\mathrm{t}, 3 \mathrm{H}, J=7.6 \mathrm{~Hz}) ; 1.36-1.45(\mathrm{~m}, 2 \mathrm{H}) ; 1.66-$ $1.75(\mathrm{~m}, 2 \mathrm{H}) ; 2.54(\mathrm{t}, 2 \mathrm{H}, J=7.6 \mathrm{~Hz}) ; 6.25(\mathrm{~s}, 1 \mathrm{H})$; $7.36(\mathrm{~d}, 1 \mathrm{H}, J=7.6 \mathrm{~Hz}) ; 7.63-7.69(\mathrm{~m}, 1 \mathrm{H}) ; 8.23-$ $8.27(\mathrm{~m}, 1 \mathrm{H}) ; 7.42-7.46(\mathrm{~m}, 1 \mathrm{H}) .{ }^{13} \mathrm{C}$ NMR $(125 \mathrm{MHz}$, $\left.\mathrm{CDCl}_{3}\right): \delta_{\mathrm{C}} 13.6,22.1,29.0,37.6,102.8,122.9,127.4$, 1219.6, 130.3, 134.6, 136.0, 137.8, 158.4. MS (EI): $m / z=202\left[\mathrm{M}^{+}\right]$. Anal. Calcd for $\mathrm{C}_{13} \mathrm{H}_{14} \mathrm{O}_{2}: \mathrm{C}, 77.20$; $\mathrm{H}, 6.98 \%$. Found: C, 77.35; H, 7.02\%.

2.2e 3-Hexyl-1H-isochromen-1-one $e^{28} \quad$ (2e): Yellow oil; IR (neat): 2928, 1733, 1655, $1158 \mathrm{~cm}^{-1}$; ${ }^{1} \mathrm{H}$ NMR $\left(500 \mathrm{MHz}, \mathrm{CDCl}_{3}\right): \delta_{\mathrm{H}} 0.89(\mathrm{t}, 3 \mathrm{H}, J=6.9 \mathrm{~Hz}) ; 1.25-$ $1.40(\mathrm{~m}, 6 \mathrm{H}) ; 1.66-1.76(\mathrm{~m}, 2 \mathrm{H}) ; 2.49-2.53(\mathrm{~m}, 2 \mathrm{H})$; $6.25(\mathrm{~s}, 1 \mathrm{H}) ; 7.35(\mathrm{~d}, 1 \mathrm{H}, J=7.8 \mathrm{~Hz}) ; 7.41-7.45(\mathrm{~m}$, $1 \mathrm{H})$; 7.65-7.69 (m, $1 \mathrm{H}) ; 8.23-8.25(\mathrm{~m}, 1 \mathrm{H}) .{ }^{13} \mathrm{C}$ NMR $\left(125 \mathrm{MHz}, \mathrm{CDCl}_{3}\right): \delta_{\mathrm{C}} 13.9,23.0,28.5,29.6,30.2,38.7$, $102.8,127.6,129.3,134.5,137.5,158.3,163.1$. MS (EI): $m / z=230\left[\mathrm{M}^{+}\right]$. Anal. Calcd for $\mathrm{C}_{15} \mathrm{H}_{18} \mathrm{O}_{2}$ : 78.23; H, 7.88\%. Found: C, 78.30; H, 7.81\%.

$2.2 \mathrm{f}$ 5-Nitro-3-phenyl-1H-isochromen-1-one (2f): Yellow solid; mp $140-142^{\circ} \mathrm{C}$ (lit. ${ }^{29} \mathrm{mp} 142-143^{\circ} \mathrm{C}$ ); IR (KBr): 1349, 1522, $1744 \mathrm{~cm}^{-1}$; ${ }^{1} \mathrm{H}$ NMR $(500 \mathrm{MHz}$, $\left.\mathrm{CDCl}_{3}\right): \delta_{\mathrm{H}} 7.51-7.55(\mathrm{~m}, 3 \mathrm{H}), 7.65(\mathrm{t}, 1 \mathrm{H}, J=$ $7.6 \mathrm{~Hz}) ; 7.85(\mathrm{~d}, 1 \mathrm{H}, J=0.8 \mathrm{~Hz}) ; 7.95-8.00(\mathrm{~m}, 2 \mathrm{H})$, $8.55\left(\mathrm{dd}, 1 \mathrm{H}, J_{1}=8.2, J_{2}=1.2 \mathrm{~Hz}\right) ; 8.69(\mathrm{ddd}, 1 \mathrm{H}$, $\left.J_{1}=8.2, J_{2}=1.2, J_{3}=0.8 \mathrm{~Hz}\right) .{ }^{13} \mathrm{C} \mathrm{NMR}(125 \mathrm{MHz}$, $\left.\mathrm{CDCl}_{3}\right): \delta_{\mathrm{C}} 102.4,114.2,122.4,126.5,127.5,128.6$, 129.2, 129.3, 131.1, 136.2, 144.6, 148.9, 152.8, 164.0. MS (EI): $m / z=267\left[\mathrm{M}^{+}\right]$. Anal. Calcd for $\mathrm{C}_{15} \mathrm{H}_{9} \mathrm{NO}_{4}$ : C, 67.42; H, 3.39; N, 5.24\%. Found: C, 67.30; H, 3.41; $\mathrm{N}, 5.28 \%$.

2.2g 5-Nitro-3-p-tolyl-1H-isochromen-1-one (2g): Yellow solid: $\mathrm{mp} 159-161^{\circ} \mathrm{C}$ (lit. ${ }^{29} \mathrm{mp} 161-162^{\circ} \mathrm{C}$ ); IR (KBr): 1325, 1521, 1622, $1741 \mathrm{~cm}^{-1} ;{ }^{1} \mathrm{H}$ NMR $\left(500 \mathrm{MHz}, \mathrm{CDCl}_{3}\right): \delta_{\mathrm{H}} 2.41(\mathrm{~s}, 3 \mathrm{H}) ; 7.27(\mathrm{~d}, 2 \mathrm{H}, J=$ $8.4 \mathrm{~Hz}), 7.55$ (t, $1 \mathrm{H}, J=8.4 \mathrm{~Hz}) ; 7.79(\mathrm{~s}, 1 \mathrm{H}) ; 7.81$ $(\mathrm{d}, 2 \mathrm{H}, J=8.4 \mathrm{~Hz}) ; 8.49\left(\mathrm{dd}, 1 \mathrm{H}, J_{1}=8.2, J_{2}=\right.$ $1.3 \mathrm{~Hz}) ; 8.63\left(\mathrm{dt}, 1 \mathrm{H}, J_{1}=8.2, J_{2}=1.2 \mathrm{~Hz}\right) .{ }^{13} \mathrm{C}$ NMR $\left(125 \mathrm{MHz}, \mathrm{CDCl}_{3}\right): \delta_{\mathrm{C}} 20.9,102.0,113.8,122.5$, 126.2, 127.3, 128.9, 129.1, 131.0, 136.0, 144.5, 148.7, 152.3, 164.1. MS (EI): $m / z=281\left[\mathrm{M}^{+}\right]$. Anal. Calcd 
for $\mathrm{C}_{16} \mathrm{H}_{11} \mathrm{NO}_{4}: \mathrm{C}, 68.32 ; \mathrm{H}, 3.94 ; \mathrm{N}, 4.98 \%$. Found: $\mathrm{C}$, $68.20 ; \mathrm{H}, 3.97 ; \mathrm{N}, 5.02 \%$.

2.2h 3-(4-Methoxyphenyl)-5-nitro-1H-isochromen-1one $(\mathbf{2 h})$ : Yellow solid: $\mathrm{mp} 238-240^{\circ} \mathrm{C}$ (lit. ${ }^{29} \mathrm{mp}$ 241-242 ${ }^{\circ} \mathrm{C}$ ); IR (KBr): $1350,1517,1625,1732 \mathrm{~cm}^{-1}$; ${ }^{1} \mathrm{H}$ NMR $\left(500 \mathrm{MHz}, \mathrm{CDCl}_{3}\right): \delta_{\mathrm{H}} 3.89(\mathrm{~s}, 3 \mathrm{H}) ; 6.97(\mathrm{~d}$, $2 \mathrm{H}, J=9.1 \mathrm{~Hz}), 7.55(\mathrm{t}, 1 \mathrm{H}, J=8.4 \mathrm{~Hz}) ; 7.77(\mathrm{~s}$, $1 \mathrm{H}) ; 7.90(\mathrm{~d}, 2 \mathrm{H}, J=9.1 \mathrm{~Hz}) ; 8.48\left(\mathrm{dd}, 1 \mathrm{H}, J_{1}=8.4\right.$, $\left.J_{2}=1.3 \mathrm{~Hz}\right) ; 8.61\left(\mathrm{dd}, 1 \mathrm{H}, J_{1}=8.4, J_{2}=1.3 \mathrm{~Hz}\right)$. ${ }^{13} \mathrm{C}$ NMR $\left(125 \mathrm{MHz}, \mathrm{CDCl}_{3}\right): \delta_{\mathrm{C}} 55.8,102.2,114.0$, $122.3,126.0,127.1,128.5,129.5,131.4,136.7,143.9$, 149.9, 161.4, 164.6. MS (EI): $m / z=297\left[\mathrm{M}^{+}\right]$. Anal. Calcd for $\mathrm{C}_{16} \mathrm{H}_{11} \mathrm{NO}_{5}$ : C, 64.65; H, 3.73; N, $4.71 \%$. Found: C, 64.50; H, 3.75; N, 4.77\%.

2.2i 6-Methoxy-3-phenyl-1H-isochromen-1-one (2i): Colourless solid; mp $135-137^{\circ} \mathrm{C}$ (lit. ${ }^{30} 136.5-$ $137.0^{\circ} \mathrm{C}$ ); IR (KBr): $1732,1607,1519,720 \mathrm{~cm}^{-1} .{ }^{1} \mathrm{H}$ NMR $\left(500 \mathrm{MHz}, \mathrm{CDCl}_{3}\right): \delta_{\mathrm{H}} 3.94(\mathrm{~s}, 3 \mathrm{H}) ; 6.90(\mathrm{~d}$, $1 \mathrm{H}, J=2.6 \mathrm{~Hz}) ; 6.92(\mathrm{~s}, 1 \mathrm{H}) ; 7.05(\mathrm{dd}, 1 \mathrm{H}, J=2.6$, $8.6 \mathrm{~Hz}) ; 7.43-7.48(\mathrm{~m}, 3 \mathrm{H}) ; 7.85(\mathrm{dd}, 2 \mathrm{H}, J=1.3$, $8.6 \mathrm{~Hz}) ; 8.25(\mathrm{~d}, 1 \mathrm{H}, J=8.6 \mathrm{~Hz}) .{ }^{13} \mathrm{C} \mathrm{NMR}(125 \mathrm{MHz}$, $\left.\mathrm{CDCl}_{3}\right): \delta_{\mathrm{C}} 56.5,101.9,108.9,109.9,118.0,125.0$, $125.7,128.9,129.3,130.5,131.6,136.7,141.2,155.1$, 159.9, 162.5. MS (EI): $m / z=252\left[\mathrm{M}^{+}\right]$. Anal. Calcd for $\mathrm{C}_{16} \mathrm{H}_{12} \mathrm{O}_{3}$ : C, 76.18; $\mathrm{H}, 4.79 \%$. Found: C, 75.99; H, $4.84 \%$.

$2.2 \mathrm{j}$ 6-Methoxy-3-(4-methoxyphenyl)-1H-isochromenl-one (2j): Colourless solid; $\mathrm{mp} 144-146^{\circ} \mathrm{C}$ (lit. ${ }^{31}$ mp 145-147 $\left.{ }^{\circ} \mathrm{C}\right)$; IR (KBr): $1737,1605,1514, \mathrm{~cm}^{-1}$. ${ }^{1} \mathrm{H}$ NMR $\left(500 \mathrm{MHz}, \mathrm{CDCl}_{3}\right): \delta_{\mathrm{H}} 3.87$ (s, 3H); 3.93 (s, $3 \mathrm{H}) ; 6.75(\mathrm{~s}, 1 \mathrm{H}) ; 6.83(\mathrm{~d}, 1 \mathrm{H}, J=2.6 \mathrm{~Hz}) ; 6.90-7.00$ $(\mathrm{m}, 2 \mathrm{H}) ; 7.02\left(\mathrm{dd}, 1 \mathrm{H}, J_{1}=8.6, J_{2}=2.6 \mathrm{~Hz}\right) ; 7.75-$ $7.88(\mathrm{~m}, 2 \mathrm{H}) ; 8.20(\mathrm{~d}, 1 \mathrm{H}, J=8.8 \mathrm{~Hz}) \cdot{ }^{13} \mathrm{C} \mathrm{NMR}$ $\left(125 \mathrm{MHz}, \mathrm{CDCl}_{3}\right): \delta_{\mathrm{C}} 55.4,55.5,100.1,107.4,113.3$, 114.1, 114.2, 116.0, 124.4, 126.7, 126.8, 131.7, 140.0, 154.1, 161.0, 162.1, 164.3. MS (EI): $m / z=282\left[\mathrm{M}^{+}\right]$. Anal. calcd for $\mathrm{C}_{17} \mathrm{H}_{14} \mathrm{O}_{4}: \mathrm{C}, 72.33 ; \mathrm{H}, 5.00 \%$. Found: C, $72.45 ; \mathrm{H}, 4.97 \%$.

2.2k 8-Methoxy-3-phenyl-1H-isochromen-1-one (2k): Yellow solid; $\mathrm{mp} 142-144^{\circ} \mathrm{C}$ (lit. ${ }^{32} \mathrm{mp} 143-145^{\circ} \mathrm{C}$ ); IR (KBr): $1723,1569,1480 \mathrm{~cm}^{-1} \cdot{ }^{1} \mathrm{H}$ NMR $(500 \mathrm{MHz}$, $\left.\mathrm{CDCl}_{3}\right): \delta_{\mathrm{H}} 4.02(\mathrm{~s}, 3 \mathrm{H}) ; 6.85(\mathrm{~s}, 1 \mathrm{H}) ; 6.95(\mathrm{~d}, 1 \mathrm{H}$, $J=8.0 \mathrm{~Hz}) ; 7.05(\mathrm{~d}, 1 \mathrm{H}, J=8.1 \mathrm{~Hz}) ; 7.40-7.54(\mathrm{~m}$, $3 \mathrm{H}) ; 7.65(\mathrm{t}, 1 \mathrm{H}, J=8.0 \mathrm{~Hz}) ; 7.82-7.95(\mathrm{~m}, 2 \mathrm{H}) .{ }^{13} \mathrm{C}$ NMR $\left(125 \mathrm{MHz}, \mathrm{CDCl}_{3}\right): \delta_{\mathrm{C}} 56.4,101.7,109.1,109.9$, 118.1, 125.2, 125.3, 128.6, 128.7, 129.8, 131.6, 135.6, 140.5, 153.8, 159.0, 161.7. MS (EI): $m / z=252\left[\mathrm{M}^{+}\right]$.
Anal. Calcd for $\mathrm{C}_{16} \mathrm{H}_{12} \mathrm{O}_{3}: \mathrm{C}, 76.18 ; \mathrm{H}, 4.79 \%$. Found: C, $76.30 ; \mathrm{H}, 4.75 \%$.

2.21 3-Ethyl-6-methoxy-1H-isochromen-1-one (2l): Colourless solid; mp 90-92 ${ }^{\circ} \mathrm{C}$ (lit. ${ }^{33} \mathrm{mp} 93-95^{\circ} \mathrm{C}$ ); IR (KBr): 3085, 2992, 1728, 1647, 1605, $1155 \mathrm{~cm}^{-1} .{ }^{1} \mathrm{H}$ NMR $\left(500 \mathrm{MHz}, \mathrm{CDCl}_{3}\right): \delta_{\mathrm{H}} 1.33(\mathrm{t}, 3 \mathrm{H}, J=7.6 \mathrm{~Hz})$; $2.61(\mathrm{q}, 2 \mathrm{H}, J=7.6 \mathrm{~Hz}) ; 3.95(\mathrm{~s}, 3 \mathrm{H}) ; 6.25(\mathrm{~s}, 1 \mathrm{H})$; $6.79(\mathrm{~d}, 1 \mathrm{H}, J=2.6 \mathrm{~Hz}) ; 7.03\left(\mathrm{dd}, 1 \mathrm{H}, J_{1}=8.6, J_{2}=\right.$ $2.6 \mathrm{~Hz}) ; 8.22(\mathrm{~d}, 1 \mathrm{H}, J=8.6 \mathrm{~Hz}) .{ }^{13} \mathrm{C} \mathrm{NMR}(125 \mathrm{MHz}$, $\left.\mathrm{CDCl}_{3}\right): \delta_{\mathrm{C}} 11.5,27.0,55.9,102.5,107.5,113.6,116.4$, 132.1, 140.2, 160.5, 163.2, 165.2. MS (EI): $m / z=204$ $\left[\mathrm{M}^{+}\right]$. Anal. Calcd for $\mathrm{C}_{12} \mathrm{H}_{12} \mathrm{O}_{3}: \mathrm{C}, 70.57 ; \mathrm{H}, 5.92 \%$. Found: C, 70.30; H, 5.98\%.

$2.2 \mathrm{~m}$ 6-Methoxy-3-methyl-1H-isochromen-1-one (2m): Colourless solid; mp $85-87^{\circ} \mathrm{C}$ (lit. ${ }^{34} \mathrm{mp} 86-88^{\circ} \mathrm{C}$ ); IR (KBr): 3075, 2998, 1725, 1650, 1614, $1144 \mathrm{~cm}^{-1} .{ }^{1} \mathrm{H}$ NMR $\left(500 \mathrm{MHz}, \mathrm{CDCl}_{3}\right): \delta_{\mathrm{H}} 2.32(\mathrm{~s}, 3 \mathrm{H}), 3.95(\mathrm{~s}, 3 \mathrm{H})$, $6.24(\mathrm{~s}, 1 \mathrm{H}), 6.76(\mathrm{~d}, 1 \mathrm{H}, J=2.6 \mathrm{~Hz}), 7.02(1 \mathrm{H}, \mathrm{dd}$, $\left.J_{1}=8.6, J_{2}=2.6 \mathrm{~Hz}, 1 \mathrm{H}\right), 8.20(\mathrm{~d}, 1 \mathrm{H}, J=8.6 \mathrm{~Hz}$, $1 \mathrm{H}) ; 13 \mathrm{C}$ NMR $\left(125 \mathrm{MHz}, \mathrm{CDCl}_{3}\right): \delta_{\mathrm{C}} 20.1,56.1$, 104.1, 107.3, 113.4, 116.2, 132.1, 140.3, 155.5, 163.1, 165.0. MS (EI): $m / z=190\left[\mathrm{M}^{+}\right]$. Anal. Calcd for $\mathrm{C}_{11} \mathrm{H}_{10} \mathrm{O}_{3}$ : C, 69.46; H, 5.30\%. Found: C, 69.60; H, $5.26 \%$.

2.2n 8-Fluoro-3-ethyl-1H-isochromen-1-one33 (2n): Colourless oil; IR (neat): 3083, 2973, 1743, 1661, $1614 \mathrm{~cm}^{-1} ;{ }^{1} \mathrm{H}$ NMR $\left(500 \mathrm{MHz}, \mathrm{CDCl}_{3}\right): \delta_{\mathrm{H}} 1.30(\mathrm{t}, 3 \mathrm{H}$, $J=7.6 \mathrm{~Hz}) ; 2.59\left(\mathrm{qd}, 2 \mathrm{H}, J_{1}=7.6, J_{2}=1.3 \mathrm{~Hz}\right) ; 6.25$ $(\mathrm{t}, 1 \mathrm{H}, J=1.3 \mathrm{~Hz}) ; 7.05-7.17(\mathrm{~m}, 2 \mathrm{H}) ; 7.60-7.72(\mathrm{~m}$, $1 \mathrm{H}) ;{ }^{13} \mathrm{C}$ NMR $\left(125 \mathrm{MHz}, \mathrm{CDCl}_{3}\right): \delta_{\mathrm{C}} 11.4,27.1,102.0$ $\left(\mathrm{d}, J_{\mathrm{C}-\mathrm{F}}=3.1 \mathrm{~Hz}\right), 109.5\left(\mathrm{~d}, J_{\mathrm{C}-\mathrm{F}}=7.1 \mathrm{~Hz}\right), 114.9$ $\left(\mathrm{d}, J_{\mathrm{C}-\mathrm{F}}=21.0 \mathrm{~Hz}\right), 121.5\left(\mathrm{~d}, J_{\mathrm{C}-\mathrm{F}}=4.1 \mathrm{~Hz}\right), 136.7$ $\left(\mathrm{d}, J_{\mathrm{C}-\mathrm{F}}=10.0 \mathrm{~Hz}\right), 140.5,158.9\left(\mathrm{~d}, J_{\mathrm{C}-\mathrm{F}}=5.6 \mathrm{~Hz}\right)$, $161.2,163.1\left(\mathrm{~d}, J_{\mathrm{C}-\mathrm{F}}=266.1 \mathrm{~Hz}\right)$. MS (EI): $\mathrm{m} / z=192$ $\left[\mathrm{M}^{+}\right]$. Anal. Calcd for $\mathrm{C}_{11} \mathrm{H}_{9} \mathrm{FO}_{2}: \mathrm{C}, 68.74 ; \mathrm{H}, 4.72 \%$. Found: C, 68.90; H, 4.66\%.

$2.20 \quad 3-P h e n y l-1 H$-benzo[g]isochromen-1-one (2o): Brown solid; mp $182-183^{\circ} \mathrm{C}$; (lit. ${ }^{35} \mathrm{mp} 180.5-181^{\circ} \mathrm{C}$ ); IR (KBr): $1730,1629 \mathrm{~cm}^{-1}$. ${ }^{1} \mathrm{H}$ NMR $(500 \mathrm{MHz}$, $\left.\mathrm{CDCl}_{3}\right): \delta_{\mathrm{H}} 7.06(\mathrm{~s}, 1 \mathrm{H}) ; 7.40-7.50(\mathrm{~m}, 3 \mathrm{H}) ; 7.56(\mathrm{t}$, $1 \mathrm{H}, J=7.6 \mathrm{~Hz}) ; 7.67(\mathrm{t}, 1 \mathrm{H}, J=7.6 \mathrm{~Hz}) ; 7.91-7.92$ $(\mathrm{m}, 4 \mathrm{H}) ; 8.02(\mathrm{~d}, 1 \mathrm{H}, J=8.3 \mathrm{~Hz}) ; 8.93(\mathrm{~s}, 1 \mathrm{H}) .{ }^{13} \mathrm{C}$ NMR $\left(125 \mathrm{MHz}, \mathrm{CDCl}_{3}\right): \delta_{\mathrm{C}} 101.9,119.0,124.3$, 125.1, 126.7, 127.7, 128.8, 129.4, 129.8, 129.8, 132.0, 132.1, 132.2, 132.4, 136.6, 152.0, 162.6. MS (EI): $m / z=272\left[\mathrm{M}^{+}\right]$. Anal. Calcd for $\mathrm{C}_{19} \mathrm{H}_{12} \mathrm{O}_{2}: \mathrm{C}, 83.81$; H, 4.44\%. Found: C, 83.45; H, 4.57\%. 
$2.2 p \quad 3$-Butyl-1H-benzo[g]isochromen-1-one (2p): Pale yellow solid; mp $103-105^{\circ} \mathrm{C}$; IR $(\mathrm{KBr})$ : 1728 , $1625 \mathrm{~cm}^{-1} .{ }^{1} \mathrm{H}$ NMR $\left(500 \mathrm{MHz}, \mathrm{CDCl}_{3}\right): \delta_{\mathrm{H}} 0.95(\mathrm{t}$, $3 \mathrm{H}, J=6.9 \mathrm{~Hz}) ; 1.32-1.34(\mathrm{~m}, 2 \mathrm{H}) ; 1.59-1.61(\mathrm{~m}$, $2 \mathrm{H}) ; 2.55(\mathrm{t}, 2 \mathrm{H}, J=6.9 \mathrm{~Hz}) ; 6.58(\mathrm{~s}, 1 \mathrm{H}) ; 7.33(\mathrm{~d}, 1 \mathrm{H}$, $J=7.6 \mathrm{~Hz}) ; 7.52-7.62(\mathrm{~m}, 1 \mathrm{H}) ; 7.77-7.86(\mathrm{~m}, 1 \mathrm{H})$; $7.97-8.06(\mathrm{~m}, 1 \mathrm{H}) ; 8.12(\mathrm{~s}, 1 \mathrm{H}) ; 8.49(\mathrm{~s}, 1 \mathrm{H}) .{ }^{13} \mathrm{C}$ NMR $\left(125 \mathrm{MHz}, \mathrm{CDCl}_{3}\right): \delta_{\mathrm{C}} 13.8,22.3,29.1,37.9$, 102.0, 125.2, 125.3, 126.5, 127.4, 128.5, 129.0, 129.3, 131.5, 134.0, 134.9, 151.8, 162.2. MS (EI): $m / z=252$ $\left[\mathrm{M}^{+}\right]$. Anal. Calcd for $\mathrm{C}_{17} \mathrm{H}_{16} \mathrm{O}_{2}: \mathrm{C}, 80.93 ; \mathrm{H}, 6.39 \%$. Found: C, 81.04; $\mathrm{H}, 6.35 \%$.

$2.2 q$ 3-p-Tolyl-1H-benzo[g]isochromen-1-one (2q): Yellow solid; mp $159-161^{\circ} \mathrm{C}$; IR (KBr): 1729, $1626 \mathrm{~cm}^{-1} \cdot{ }^{1} \mathrm{H}$ NMR $\left(500 \mathrm{MHz}, \mathrm{CDCl}_{3}\right): \delta_{\mathrm{H}} 2.37(\mathrm{~s}$, $3 \mathrm{H}) ; 7.01(\mathrm{~s}, 1 \mathrm{H}) ; 7.09(\mathrm{~d}, 2 \mathrm{H}, J=8.4 \mathrm{~Hz}) ; 7.27$ (d, $2 \mathrm{H}, J=8.4 \mathrm{~Hz}) ; 7.34-7.45(\mathrm{~m}, 4 \mathrm{H}) ; 7.97(\mathrm{~s}, 1 \mathrm{H}) ; 8.89$ (s, $1 \mathrm{H}) .{ }^{13} \mathrm{C}$ NMR $\left(125 \mathrm{MHz}, \mathrm{CDCl}_{3}\right): \delta_{\mathrm{C}} 22.9,100.2$, $119.0,124.9,125.0,126.1,126.3,126.5,127.0,127.2$, 128.1, 129.0, 129.1, 131.3, 134.0, 134.9, 151.7, 162.7. MS (EI): $m / z=286\left[\mathrm{M}^{+}\right]$. Anal. Calcd for $\mathrm{C}_{20} \mathrm{H}_{14} \mathrm{O}_{2}$ : C, 83.90; H, 4.93\%. Found: C, 84.05; H, 4.89\%.

2.2r 3-o-Tolyl-1H-benzo[g]isochromen-1-one (2r): Yellow solid; mp $166-168^{\circ} \mathrm{C}$; IR $(\mathrm{KBr})$ : 1728 , $1625 \mathrm{~cm}^{-1} .{ }^{1} \mathrm{H}$ NMR $\left(500 \mathrm{MHz}, \mathrm{CDCl}_{3}\right): \delta_{\mathrm{H}} 2.35(\mathrm{~s}$, $3 \mathrm{H}) ; 7.05(\mathrm{~s}, 1 \mathrm{H}) ; 7.26-7.40(\mathrm{~m}, 4 \mathrm{H}) ; 7.53-7.72(\mathrm{~m}$, $4 \mathrm{H}) ; 8.10(\mathrm{~s}, 1 \mathrm{H}) ; 8.74(\mathrm{~s}, 1 \mathrm{H}) .{ }^{13} \mathrm{C}$ NMR $(125 \mathrm{MHz}$, $\left.\mathrm{CDCl}_{3}\right): \delta_{\mathrm{C}} 22.7,100.3,119.1,121.0,122.4,124.7$, $125.1,126.2,126.1,126.7,127.0,127.3,128.2,129.0$, $129.7,131.5,134.2,134.9,151.6,162.5$. MS (EI): $m / z=286\left[\mathrm{M}^{+}\right]$. Anal. Calcd for $\mathrm{C}_{19} \mathrm{H}_{12} \mathrm{O}_{2}: \mathrm{C}, 83.90$; H, 4.93\%. Found: C, 83.65; H, 4.99\%.

2.2s 7-Phenyl-5H-[1,3]dioxolo[4,5-g]isochromen-5one (2s): Pale yellow solid; $\mathrm{mp} 113-115^{\circ} \mathrm{C}$; $\mathrm{IR}(\mathrm{KBr})$ : 1738, 1630, 1034, $755 \mathrm{~cm}^{-1}$. ${ }^{1} \mathrm{H}$ NMR $(500 \mathrm{MHz}$, $\left.\mathrm{CDCl}_{3}\right): \delta_{\mathrm{H}} 5.91(\mathrm{~s}, 2 \mathrm{H}) ; 6.71(\mathrm{~s}, 1 \mathrm{H}) ; 7.04(\mathrm{~s}, 1 \mathrm{H})$; 7.15-7.24 (m, 3H); 7.29 (s, 1H); 7.33-7.39 (m, 2H). ${ }^{13} \mathrm{C}$ NMR $\left(125 \mathrm{MHz}, \mathrm{CDCl}_{3}\right): \delta_{\mathrm{C}} 98.5,101.4,111.1$, 113.2, 122.4, 125.9, 128.1, 128.9, 131.2, 131.9, 145.1, 148.1, 154.0, 163.2. MS (EI): $m / z=266\left[\mathrm{M}^{+}\right]$. Anal. Calcd for $\mathrm{C}_{16} \mathrm{H}_{10} \mathrm{O}_{4}$ : C, 72.18; H, 3.79\%. Found: C, $72.02 ; \mathrm{H}, 3.84 \%$.

2.2t 7-Butyl-5H-[1,3]dioxolo[4,5-g]isochromen-5one (2t): Colourless solid; mp 97-99 ${ }^{\circ} \mathrm{C}$; IR (KBr): 1733, 1627, 1035, $757 \mathrm{~cm}^{-1} \cdot{ }^{1} \mathrm{H}$ NMR $(500 \mathrm{MHz}$, $\left.\mathrm{CDCl}_{3}\right): \delta_{\mathrm{H}} 0.96(\mathrm{t}, 3 \mathrm{H}, J=6.9 \mathrm{~Hz}) ; 1.33-1.36(\mathrm{~m}$, $2 \mathrm{H}) ; 1.54-1.62(\mathrm{~m}, 2 \mathrm{H}) ; 2.10(\mathrm{t}, 2 \mathrm{H}, J=6.9 \mathrm{~Hz}) ; 5.90$ $(\mathrm{s}, 2 \mathrm{H}) ; 6.13(\mathrm{~s}, 1 \mathrm{H}) ; 6.98(\mathrm{~s}, 1 \mathrm{H}) ; 7.51(\mathrm{~s}, 1 \mathrm{H}) \cdot{ }^{13} \mathrm{C}$ NMR $\left(125 \mathrm{MHz}, \mathrm{CDCl}_{3}\right): \delta_{\mathrm{C}} 13.9,22.8,29.1,36.9$, $101.9,102.3,117.1,121.0,122.9,132.2,148.2,151.1$, 154.6, 162.0. MS (EI): $m / z=246\left[\mathrm{M}^{+}\right]$. Anal. Calcd for $\mathrm{C}_{19} \mathrm{H}_{12} \mathrm{O}_{2}$ : C, 68.28; $\mathrm{H}, 5.73 \%$. Found: $\mathrm{C}, 68.45 ; \mathrm{H}$, $5.69 \%$.

$2.2 \mathrm{u}$ 7-p-Tolyl-5H-[1,3]dioxolo[4,5-g]isochromen-5one $(2 u)$ : Yellow solid; $\mathrm{mp} 134-136^{\circ} \mathrm{C}$; IR $(\mathrm{KBr})$ : 1735, 1632, 1039, $760 \mathrm{~cm}^{-1}$. ${ }^{1} \mathrm{H}$ NMR $(500 \mathrm{MHz}$, $\left.\mathrm{CDCl}_{3}\right): \delta_{\mathrm{H}} 2.36(\mathrm{~s}, 3 \mathrm{H}) ; 5.93(\mathrm{~s}, 2 \mathrm{H}) ; 6.71(\mathrm{~s}, 1 \mathrm{H}) ; 7.10$ $(\mathrm{d}, 2 \mathrm{H}, J=7.6 \mathrm{~Hz}) ; 7.13(\mathrm{~s}, 1 \mathrm{H}) ; 7.32(\mathrm{~s}, 1 \mathrm{H}) ; 7.39$ $(\mathrm{d}, 2 \mathrm{H}, J=7.6 \mathrm{~Hz}) ;{ }^{13} \mathrm{C}$ NMR $\left(125 \mathrm{MHz}, \mathrm{CDCl}_{3}\right): \delta_{\mathrm{C}}$ $25.1,99.0,102.3,110.9,113.0,122.1,125.6,127.9$, $129.4,131.7,132.3,144.7,147.7,153.8,163.1 . \mathrm{MS}$ (EI): $m / z=280\left[\mathrm{M}^{+}\right]$. Anal. Calcd for $\mathrm{C}_{17} \mathrm{H}_{12} \mathrm{O}_{4}: \mathrm{C}$, 72.85; H, 4.32\%. Found: C, 73.01; H, 4.25\%.

$2.2 \mathrm{v}$ 3-Phenylpyrano[3,4-b]indol-1(9H)-one $(2 v)$ : Yellow solid; mp $171-173^{\circ} \mathrm{C}$; IR (KBr): 3401,2921 , $1686,742 \mathrm{~cm}^{-1} \cdot{ }^{1} \mathrm{H}$ NMR $\left(500 \mathrm{MHz}, \mathrm{CDCl}_{3}+\right.$ DMSO$\left.d_{6}\right): \delta_{\mathrm{H}} 7.13(\mathrm{t}, 1 \mathrm{H}, J=6.8 \mathrm{~Hz}) ; 7.17-7.19(\mathrm{~m}, 1 \mathrm{H})$; $7.27(\mathrm{~d}, 1 \mathrm{H}, J=6.8 \mathrm{~Hz}) ; 7.34-7.37(\mathrm{~m}, 3 \mathrm{H}) ; 7.39$ (s, $1 \mathrm{H}) ; 7.45-7.50(\mathrm{~m}, 1 \mathrm{H}) ; 7.79(\mathrm{~d}, 1 \mathrm{H}, J=7.6 \mathrm{~Hz})$; $7.84(\mathrm{~d}, 1 \mathrm{H}, J=7.6 \mathrm{~Hz}) ; 11.94(\mathrm{~s}, 1 \mathrm{H}) .{ }^{13} \mathrm{C} \mathrm{NMR}$ $\left(125 \mathrm{MHz}, \mathrm{CDCl}_{3}+\mathrm{DMSO}-d_{6}\right): \delta_{\mathrm{C}} 97.7,113.4,120.8$, $121.4,121.9,124.3,124.8,125.6,127.7,128.9,129.0$, 132.8, 140.6, 151.9, 157.0. MS (EI): $m / z=261\left[\mathrm{M}^{+}\right]$. Anal. Calcd for $\mathrm{C}_{17} \mathrm{H}_{11} \mathrm{NO}_{2}$ : C, 78.15; H, 4.24; N, 5.36. Found: C, 78.39; H, 4.19; N, 5.26\%.

2.2w 9-Methyl-3-phenylpyrano[3,4-b]indol-1(9H)-one (2w): Yellow solid; Mp 168-170 ${ }^{\circ} \mathrm{C}$; IR (KBr): 1711, 1471，1222，1063， $742 \mathrm{~cm}^{-1} .{ }^{1} \mathrm{H}$ NMR $(500 \mathrm{MHz}$, DMSO- $d_{6}$, variable temperature): $\delta_{\mathrm{H}} 4.02(\mathrm{~s}, 3 \mathrm{H})$; 7.22-7.23 (m, 1H); 7.36-7.56 (m, 5H); 7.81-7.85 (m, $3 \mathrm{H}) ; 8.02-8.04(\mathrm{~m}, 1 \mathrm{H}) .{ }^{13} \mathrm{C}$ NMR $(125 \mathrm{MHz}$, DMSO$d_{6}$, variable temperature): $\delta_{\mathrm{C}} 31.6,98.3,111.6,120.9$, $121.2,121.4,122.3,124.8,125.6,128.3,129.4,129.5$, 132.6, 141.5, 151.6, 156.1. MS (EI): $m / z=275\left[\mathrm{M}^{+}\right]$. Anal. Calcd for $\mathrm{C}_{18} \mathrm{H}_{13} \mathrm{NO}_{2}$ : C, 78.53; H, 4.76; N, 5.09. Found: C, 78.69; H, 4.72; N, 5.03\%.

2.2x 9-Ethyl-3-phenylpyrano[3,4-b]indol-1(9H)-one $(2 x)$ : Orange solid; $\mathrm{mp} 70-75^{\circ} \mathrm{C}$; IR (neat): 1712 , 1469, 1220, 1061, $739 \mathrm{~cm}^{-1} .{ }^{1} \mathrm{H}$ NMR $(500 \mathrm{MHz}$, $\left.\mathrm{CDCl}_{3}\right): \delta_{\mathrm{H}} 1.44(\mathrm{t}, 3 \mathrm{H}, J=7.6 \mathrm{~Hz}) ; 4.66(\mathrm{q}, 2 \mathrm{H}, J=$ $7.6 \mathrm{~Hz}) ; 7.24(\mathrm{t}, 1 \mathrm{H}, J=6.9 \mathrm{~Hz}) ; 7.29(\mathrm{~s}, 1 \mathrm{H}) ; 7.35$ $(\mathrm{d}, 1 \mathrm{H}, J=6.9 \mathrm{~Hz}) ; 7.41-7.43(\mathrm{~m}, 3 \mathrm{H}) ; 7.49(\mathrm{t}, 1 \mathrm{H}$, $J=6.9 \mathrm{~Hz}) ; 7.85-7.89(\mathrm{~m}, 3 \mathrm{H}) .{ }^{13} \mathrm{C}$ NMR $(125 \mathrm{MHz}$, $\left.\mathrm{CDCl}_{3}\right): \delta_{\mathrm{C}} 15.9,39.6,97.1,110.7,120.4,120.9,121.4$, 
121.7, 124.9, 125.9, 127.9, 128.8, 129.1, 132.6, 140.4, 152.4, 156.5. MS (EI): $m / z=289\left[\mathrm{M}^{+}\right]$. Anal. Calcd for $\mathrm{C}_{19} \mathrm{H}_{15} \mathrm{NO}_{2}$ : C, 78.87; H, 5.23; N, 4.84. Found: C, 78.00; H, 4.28; N, 5.41\%.

\subsection{Animals and drug dosage}

2.3a Animals: The selection of animals, caring and handling was done as per the guidelines set by the Indian National Science Academy, New Delhi, India. Inbred albino mice (Swiss strain) of adult gender weighing $120-150 \mathrm{~g}$ were used for the study. The mice were housed individually in clean polypropylene cages containing sterile paddy husk (procured locally) as bedding throughout the experiment. All animals were fed with sterile commercial pelleted rat chow supplied by Hindustan Lever Ltd (Mumbai, India) with free access to water (ad libitum) under standardized housing conditions (natural light-dark cycle, temperature $23 \pm 1{ }^{\circ} \mathrm{C}$, relative humidity $55 \pm 5 \%$ ). After 7 days of adaptation to laboratory conditions, the animals were randomly assigned to 12 experimental groups of 5 mice each. Each mouse was used only once. All tests were performed between 08:00 and 16:00 h. All efforts were made to minimize animal suffering and to use only the number of animals necessary to produce reliable scientific data. The experimental protocols and procedures listed below conformed to the Guide for the Care and Use of Laboratory Animals and approved by the Institutional Ethics Committee. Mice equivalent doses in $\mathrm{mg} / \mathrm{kg}$ body weight of clinical doses were calculated as $\mathrm{mg} / \mathrm{kg}$ body weight with the help of standard tables (Karber's method). ${ }^{36}$ The test compounds were tested in mice after 14 days of administration for their safety as per OECD guidelines. ${ }^{37}$ Experimental procedure for the evaluation of antinociceptive and antiinflammatory activities was discussed in sections 3.2 and 3.3 , respectively.

\subsection{Dose and administration of compounds}

For the antinociceptive study, the target compounds $(20 \mathrm{mg} / \mathrm{kg})$, pentazocine as reference drug $(20 \mathrm{mg} / \mathrm{kg})$ and $2 \%(\mathrm{w} / \mathrm{v})$ gum acacia $(2 \mathrm{~mL} / \mathrm{kg})$ as control were administered orally by intragastric tube. For the antiinflammatory study, the target compounds $(20 \mathrm{mg} / \mathrm{kg})$, indomethacin as reference drug $(20 \mathrm{mg} / \mathrm{kg})$ and $0.5 \%$ $(\mathrm{w} / \mathrm{v})$ tween $80(2 \mathrm{~mL} / \mathrm{kg})$ as control were administerd orally.

\subsection{Statistical analysis}

The obtained data were analysed using one-way analysis of variance (ANOVA) followed by Dunnet's mul- tiple comparison test using computerized Graph Pad Instat version 3.05 (Graph Pad software, USA). The results are presented as mean \pm Standard error of means (SEM). Differences between data sets were considered as significant when $P<0.001$.

\section{Results and discussion}

\subsection{Chemistry}

2-(Phenylethynyl)benzamide 1a, which can easily be synthesized by the Sonogashira cross-coupling of 2iodobenzamide with phenylacetylene, ${ }^{38}$ was selected as a model substrate for the optimization of the reaction conditions. Several points regarding the optimization of conversion of $\mathbf{1 a}$ to $\mathbf{2 a}$ are worth noting. (i) No effort was made to exclude air or moisture in these experiments. (ii) Toluene was chosen as the solvent, since toluene is immiscible in HOTf at room temperature and forms a top-layer that avoids the evolution of toxic fumes during addition. ${ }^{39}$ Thus, the reaction of 2(phenylethynyl)benzamide 1a with HOTf (1.0 equiv) in toluene at a microwave irradiation $(300 \mathrm{~W})$ for $30 \mathrm{~min}$ with $30 \mathrm{~s}^{\text {pulse }}{ }^{40}$ showed an excellent and clean conversion toward 3-phenylisocoumarin ${ }^{41}$ which was isolated in $80 \%$ yield after aqueous work-up followed by column chromatography. ${ }^{42}$ Decreasing the amount of HOTf from 1.0 to 0.5 equiv resulted in product formation albeit in moderate yield $(60 \%)$. Since 1.0 equiv of HOTf at a power level of $300 \mathrm{~W}$ was effective for the synthesis of isocoumarin $\mathbf{2 a}$, we were interested to apply this reaction condition for other substrates. The requisite starting materials $\mathbf{1 a}-\mathbf{n}$ were synthesized as outlined in scheme 1 and the results were presented in table 1.

Under this optimized condition all substrates underwent the reaction smoothly affording the products $\mathbf{2 a - n}$ in good to excellent yields. Substrates with both electron releasing as well as withdrawing groups were tolerated under this reaction condition. Moreover, all the substrates gave isocoumarin as the exclusive product and no phthalide regioisomer was formed. ${ }^{43}$ It was reasoned that traces of water present in triflic acid effects the hydrolysis of amide. ${ }^{44}$ Irrespective of the nature of the substrates, the cyclization follows highly selective 6-endo-dig regiochemistry, which has been previously observed by Uchiyama et al. ${ }^{9}$

In view of the pharmacological significance of isocoumarins ${ }^{45}$ and their prevalence as substructure in numerous natural products, ${ }^{46}$ we extended our study to structurally diverse acetylenic amides bearing polyaromatic (schemes 2 and 3) and heteroaromatic (scheme 4) function and the results were given in table 2. Upon 
Table 1. HOTf promoted synthesis of isocoumarins (2a-2n).

\begin{tabular}{lllllcc}
\hline Entry & \multicolumn{1}{c}{$\mathrm{R}$} & $\mathrm{R}^{1}$ & $\mathrm{R}^{2}$ & $\mathrm{R}^{3}$ & Product $^{\mathrm{a}}$ & Yield (\%) $^{\mathrm{b}}$ \\
\hline 1 & $\mathrm{Ph}$ & $\mathrm{H}$ & $\mathrm{H}$ & $\mathrm{H}$ & $\mathbf{2 a}$ & 80 \\
2 & $m$-F-ph & $\mathrm{H}$ & $\mathrm{H}$ & $\mathrm{H}$ & $\mathbf{2 b}$ & 71 \\
3 & $p$-anisyl & $\mathrm{H}$ & $\mathrm{H}$ & $\mathrm{H}$ & $\mathbf{2 c}$ & 81 \\
4 & $n$-Bu & $\mathrm{H}$ & $\mathrm{H}$ & $\mathrm{H}$ & $\mathbf{2 d}$ & 82 \\
5 & $n$-hexyl & $\mathrm{H}$ & $\mathrm{H}$ & $\mathrm{H}$ & $\mathbf{2 e}$ & 81 \\
6 & $\mathrm{Ph}$ & $\mathrm{NO}_{2}$ & $\mathrm{H}$ & $\mathrm{H}$ & $\mathbf{2 f}$ & 69 \\
7 & $p$-tolyl & $\mathrm{NO}_{2}$ & $\mathrm{H}$ & $\mathrm{H}$ & $\mathbf{2 g}$ & 70 \\
8 & $p$-anisyl & $\mathrm{NO}_{2}$ & $\mathrm{H}$ & $\mathrm{H}$ & $\mathbf{2 h}$ & 71 \\
9 & Ph & $\mathrm{H}$ & $\mathrm{MeO}$ & $\mathrm{H}$ & $\mathbf{2 i}$ & 77 \\
10 & $p$-anisyl & $\mathrm{H}$ & $\mathrm{MeO}$ & $\mathrm{H}$ & $\mathbf{2 j}$ & 83 \\
11 & $\mathrm{Ph}$ & $\mathrm{H}$ & $\mathrm{H}$ & $\mathrm{MeO}$ & $\mathbf{2 k}$ & 79 \\
12 & $\mathrm{Et}$ & $\mathrm{H}$ & $\mathrm{MeO}$ & $\mathrm{H}$ & $\mathbf{2 l}$ & 85 \\
13 & $\mathrm{Me}$ & $\mathrm{H}$ & $\mathrm{MeO}$ & $\mathrm{H}$ & $\mathbf{2 m}$ & 91 \\
14 & $\mathrm{Et}$ & $\mathrm{H}$ & $\mathrm{H}$ & $\mathrm{F}$ & $\mathbf{2 n}$ & 88 \\
\hline
\end{tabular}

${ }^{\mathrm{a}}$ All products were characterized by IR, NMR and mass spectroscopy

${ }^{\mathrm{b}}$ Isolated yield

examining the fate of a variety of 3-ethynylnaphthalene-2-carboxamides 1o-r under our reaction condition, we found that these substrates underwent the reaction smoothly providing excellent yield of products 2o-r. The high yield obtained in these substrates could be attributed to the presence of naphthalene ring which enhances the electron density of the triple bond, thus interacts with the Brø̈nsted acid with ease (entries 1-4, table 2).

The utility of our methodology is further manifested by its applicability to piperanol tethered benzamides 1s-u (scheme 3). The reaction, indeed afforded the desired isocoumarin derivatives $\mathbf{2} \mathbf{s}-\mathbf{u}$ in yields ranging from $44 \%$ to $61 \%$. The low yields in these cases $2 \mathbf{s}-\mathbf{u}$ was due to the partial decomposition of the corresponding substrates under the strong acidic conditions.
However, we were not able to isolate the decomposed products, because of their sticky nature.

Our desire for the low molecular weight indole molecules led us to briefly investigate the fate of 3(ethynyl)indole-2-carboxamides $\mathbf{1} \mathbf{v}-\mathbf{x}$ under the optimized reaction condition (scheme 4). The requisite starting materials were synthesized by standard literature procedures. ${ }^{47}$ Only modest yields were observed for these substrates compared to other examples. Nevertheless, it is noteworthy to mention that similar substrates afforded no cyclization product at all under halocyclization conditions, ${ }^{13}$ a thus highlighting the significance of our protocol.

The structure of all products was confirmed by spectral data (FTIR, ${ }^{1} \mathrm{H}$ NMR, ${ }^{13} \mathrm{C}$ NMR and EI-MS) and elemental analyses. The presence of absorption



Scheme 4. Reagents and condition: (a) 3.0 equiv. $\mathrm{SOCl}_{2}, \mathrm{Et}_{2} \mathrm{O}, 0$ to $5^{\circ} \mathrm{C}, 1 \mathrm{~h}$ then excess $\mathrm{NH}_{3}$ (aq.), $0^{\circ} \mathrm{C}$ to rt, $2 \mathrm{~h}$; (b) 5.0 equiv. $\mathrm{POCl}_{3}, 100^{\circ} \mathrm{C}, 30 \mathrm{~min}$; (c) 1.1 equiv. $\mathrm{I}_{2} / \mathrm{DMF}$, rt, $3 \mathrm{~h}$; (d) 2.0 equiv. $\mathrm{NaH}, \mathrm{DMF}, 30 \mathrm{~min}$ at $0^{\circ} \mathrm{C}$ then 3.0 equiv. $\mathrm{RX}, 0^{\circ} \mathrm{C}$ to rt, $6 \mathrm{~h}$; (e) $30 \% \mathrm{H}_{2} \mathrm{O}_{2} / 3.0$ equiv. $\mathrm{Na}_{2} \mathrm{CO}_{3}$, DMSO, $5^{\circ} \mathrm{C}$ to rt, $1 \mathrm{~h}$; (f) $5 \mathrm{~mol} \% \mathrm{Pd}\left(\mathrm{PPh}_{3} \mathrm{P}\right)_{2} \mathrm{Cl}_{2} / 5 \mathrm{~mol} \% \mathrm{CuI} / \mathrm{Et} 33 \mathrm{~N}, \mathrm{rt}, 15 \mathrm{~min}, \mathrm{~N}_{2}$ then 1.1 equiv. phenylacetylene (for substrate 1v, no $N$-alkylation was carried out, thus $\mathrm{R}=\mathrm{H}$ ); (g) 1.0 equiv. $\mathrm{TfOH}$, toluene, $300 \mathrm{~W}, 3 \mathrm{~min}$. 
Table 2. HOTf promoted synthesis fused pyranones (2o-2x).

Entry

${ }^{\mathrm{a}}$ All products were characterized by IR, ${ }^{1} \mathrm{H}$ NMR, ${ }^{13} \mathrm{C}$ NMR and Mass spectroscopy

${ }^{\mathrm{b}}$ Isolated yield

at $1686-1744 \mathrm{~cm}^{-1}$ in the IR spectra of these compounds suggested a $\delta$-lactone ring. ${ }^{1} \mathrm{H}$ NMR spectra of the compounds exhibited a sharp distinct singlet at $\delta_{\mathrm{H}}$ 6.24-7.79 characteristic of $\mathrm{C} 4-\mathrm{H}$ of isocoumarin. Had it been $\gamma$-lactone, an absorption peak between 1770 and $1800 \mathrm{~cm}^{-1}$ and singlet at $\delta_{\mathrm{H}}$ (vinylic) $5.0-7.0 \mathrm{ppm}$ would be found in their IR and ${ }^{1} \mathrm{H}$ NMR spectra, respectively. ${ }^{48} \mathrm{In}{ }^{13} \mathrm{C}$ NMR spectrum the lactone carbonyl and C4-carbon resonated at $\delta_{\mathrm{C}} 156.1-165.2$ and 97.1-104.1 ppm, respectively. All these spectral values confirmed the formation of products.

\section{Pharmacology}

\subsection{Evaluation of oral toxicity}

In the present study, all the synthesized compounds were screened for their in vivo antinociceptive and antiinflammatory activities. To begin with, the oral toxicity of the synthesized compounds was performed by acute toxic class method ${ }^{36}$ in accordance with the OECD guidelines. ${ }^{37}$ The selected adult albino rats were fasted overnight prior to the acute experimental procedure. Following the period of fasting, the animals were weighed and the test compounds were orally administered at a dose of $20 \mathrm{mg} / \mathrm{kg}$ body weight. Immediately after dosing, the animals were observed for the first $30 \mathrm{~min}$ for behavioural changes and for mortality on a daily basis for a total of 14 days. There was no change in the haematological parameters and organ weights of drug treated animals compared to control.
Histopathological examination of internal organs did not show any pathological changes. As no mortality was observed with the above dose, the $\mathrm{LD}_{50}$ value of the title compounds was expected to exceed $20 \mathrm{mg} / \mathrm{kg}$ of body weight. Toxicity assays showed that all the compounds proved to be non-toxic at the tested dose levels and well-tolerated by the experimental animals as their $\mathrm{LD}_{50}$ cut-off values $>20 \mathrm{mg} / \mathrm{kg}$ body weight. However, we believe that detailed toxicological investigations are required to elucidate their chronic activity.

\subsection{Evaluation of antinociceptive activity}

Tail immersion method was used to evaluate the antinociceptive activity in male albino rats (Swiss strain) weighing $120-150$ g. ${ }^{49}$ Initially, the animals were screened for the sensitivity test by immersing the tail of the mice gently in hot water maintained at $55 \pm$ $5^{\circ} \mathrm{C}$. The animal immersing the tail from hot water with in $5 \mathrm{~s}$ was selected for the study. The animals were divided into 3 groups of 6 each. All the test compounds and standard drug were administered orally by intragastric tube. Group I was kept as control, receiving $2 \%$ $\mathrm{w} / \mathrm{v}$ of gum acacia $(2 \mathrm{~mL} / \mathrm{kg})$ in normal saline manner. Group II was kept as standard, receiving pentazocine $(20 \mathrm{mg} / \mathrm{kg})$. Group III was kept as test, receiving synthesized compounds $(20 \mathrm{mg} / \mathrm{kg})$. After administration of drugs, the animals react by withdrawing its tail. This reaction was determined before oral feeding of the standard drug and test compounds which were recorded as zero minutes reading. The time (in seconds) to 
withdraw the tail clearly out of water was taken as the reaction time. The first reading $(0 \mathrm{~min})$ was taken immediately after the administration of the test compound and subsequent reaction time was recorded at 30,60, 90 and $120 \mathrm{~min}$, respectively. The mean reaction time was recorded for each group and compared with the value of the standard drug pentazocine. The percentage antinociceptive activity was calculated using the formula: \% Antinociceptive activity $=\left[\left(\mathrm{T}_{2}-\mathrm{T}_{1}\right) / \mathrm{T}_{2}\right] \times$ 100 , where $T_{1}$ is the reaction time (in s) before treatment and $\mathrm{T}_{2}$ is the reaction time (in s) after treatment. The mean value \pm SEM was calculated for each parameter. The results were analysed statistically by ANOVA is followed by Dunnet's test. The results of the experiment by proper statistical analysis are tabulated in table 3, which revealed that all the tested compounds showed significant potency (44.24\% to $83.16 \%$ ) as compared to the standard drug pentazocine (84.81\%) at $120 \mathrm{~min}$. Among the isocoumarins, compounds having 3-hexyl $\mathbf{2 e}$ and 3-anisyl, 4-MeO substituents $\mathbf{2 j}$ showed enhanced activity compared to other derivatives of the same series. A markedly improved potency was observed when a lipophilic naphthalene system was fused with the pyranone ring 20-r. Replacement of naphthalene ring by a piperanal ring $2 \mathbf{s}-\mathbf{u}$ resulted in the significant decrease of activity. It was observed that pyranoindolones $\mathbf{2} \mathbf{v}-\mathbf{x}$ showed highest activity with values comparable to or slightly lesser than the standard pentazocine. Nevertheless, it is worth to mention that other compounds exhibited moderate to good antinociceptive activity.

\subsection{Evaluation of antiinflammatory activity}

The carrageenan-induced paw edema model in rats is known to be sensitive to cyclooxygenase inhibitors and has been used to evaluate the effect of non-steroidal antiinflammatory agents, which primarily inhibit the cyclooxygenase involved in prostaglandin synthesis. ${ }^{50}$ In the present study, the antiinflammatory activity was determined by carrageenan-induced rat paw edema method in male albino rats (Swiss strain) weighing $120-150$ g. ${ }^{51}$ The animals were divided into 3 groups of 6 each. All the test compounds and reference drug were administered orally, suspended in $0.5 \%$ aqueous solution of Tween 80 (polyoxyethylene sorbitan mononucleate) solution. Group I was kept as control,

Table 3. Antinoceceptive activity of synthesized compounds by tail immersion test.

\begin{tabular}{|c|c|c|c|c|c|c|c|c|c|c|c|}
\hline \multirow[b]{2}{*}{ Entry } & \multirow[b]{2}{*}{ Treatments } & \multirow{2}{*}{$\begin{array}{l}\text { Dose } \\
\text { levels }\end{array}$} & \multicolumn{5}{|c|}{ Tail immersion response in seconds (mean \pm SEM) } & \multicolumn{4}{|c|}{$\%$ Antinoceceptive activity } \\
\hline & & & $0 \mathrm{~min}$ & $30 \mathrm{~min}$ & $60 \mathrm{~min}$ & $90 \mathrm{~min}$ & $120 \mathrm{~min}$ & $30 \mathrm{~min}$ & $60 \mathrm{~min}$ & $90 \mathrm{~min}$ & $120 \mathrm{~min}$ \\
\hline 1 & $2 \mathbf{a}^{* *}$ & $20 \mathrm{mg} / \mathrm{kg}$ & $2.98 \pm 0.02$ & $2.04 \pm 0.01$ & $1.80 \pm 0.01$ & $1.59 \pm 0.01$ & $1.28 \pm 0.01$ & 32.89 & 40.78 & 47.52 & 57.75 \\
\hline 2 & $2 \mathbf{b}^{*}$ & $20 \mathrm{mg} / \mathrm{kg}$ & $2.99 \pm 0.01$ & $2.09 \pm 0.01$ & $1.93 \pm 0.01$ & $1.77 \pm 0.01$ & $1.60 \pm 0.01$ & 31.25 & 36.51 & 41.58 & 47.19 \\
\hline 3 & $2 \mathrm{c}^{* * *}$ & $20 \mathrm{mg} / \mathrm{kg}$ & $2.99 \pm 0.01$ & $2.03 \pm 0.02$ & $1.77 \pm 0.01$ & $1.57 \pm 0.01$ & $1.26 \pm 0.01$ & 33.22 & 41.77 & 48.18 & 58.41 \\
\hline 4 & $2 d^{*}$ & $20 \mathrm{mg} / \mathrm{kg}$ & $3.00 \pm 0.02$ & $2.01 \pm 0.01$ & $1.72 \pm 0.01$ & $1.51 \pm 0.02$ & $1.22 \pm 0.01$ & 33.88 & 43.42 & 50.16 & 59.73 \\
\hline 5 & $2 \mathrm{e}^{* *}$ & $20 \mathrm{mg} / \mathrm{kg}$ & $2.98 \pm 0.03$ & $1.98 \pm 0.01$ & $1.69 \pm 0.01$ & $1.48 \pm 0.02$ & $1.20 \pm 0.01$ & 34.86 & 44.40 & 51.15 & 60.39 \\
\hline 6 & $2 \mathrm{f} * *$ & $20 \mathrm{mg} / \mathrm{kg}$ & $2.99 \pm 0.01$ & $2.13 \pm 0.01$ & $1.93 \pm 0.01$ & $1.77 \pm 0.01$ & $1.61 \pm 0.01$ & 30.03 & 36.51 & 41.58 & 46.86 \\
\hline 7 & $2 \mathrm{~g} * *$ & $20 \mathrm{mg} / \mathrm{kg}$ & $2.98 \pm 0.01$ & $2.16 \pm 0.02$ & $1.97 \pm 0.01$ & $1.80 \pm 0.01$ & $1.64 \pm 0.01$ & 28.94 & 35.19 & 40.59 & 45.87 \\
\hline 8 & $2 \mathbf{h}^{* *}$ & $20 \mathrm{mg} / \mathrm{kg}$ & $2.98 \pm 0.01$ & $2.20 \pm 0.01$ & $2.00 \pm 0.01$ & $1.85 \pm 0.03$ & $1.69 \pm 0.01$ & 27.63 & 34.21 & 38.94 & 44.24 \\
\hline 9 & $2 i^{*}$ & $20 \mathrm{mg} / \mathrm{kg}$ & $2.99 \pm 0.01$ & $2.01 \pm 0.02$ & $1.76 \pm 0.01$ & $1.55 \pm 0.01$ & $1.24 \pm 0.01$ & 33.88 & 42.10 & 48.84 & 59.07 \\
\hline 10 & $2 \mathrm{j} * *$ & $20 \mathrm{mg} / \mathrm{kg}$ & $2.97 \pm 0.02$ & $1.99 \pm 0.01$ & $1.67 \pm 0.01$ & $1.47 \pm 0.01$ & $1.19 \pm 0.02$ & 34.53 & 45.06 & 51.48 & 60.72 \\
\hline 11 & $2 \mathbf{k}^{* *}$ & $20 \mathrm{mg} / \mathrm{kg}$ & $2.98 \pm 0.02$ & $2.02 \pm 0.02$ & $1.78 \pm 0.01$ & $1.58 \pm 0.01$ & $1.25 \pm 0.01$ & 33.55 & 41.44 & 47.85 & 58.74 \\
\hline 12 & $21 * *$ & $20 \mathrm{mg} / \mathrm{kg}$ & $2.98 \pm 0.02$ & $2.02 \pm 0.01$ & $1.74 \pm 0.01$ & $1.52 \pm 0.02$ & $1.23 \pm 0.01$ & 33.55 & 42.76 & 49.83 & 59.40 \\
\hline 13 & $2 \mathrm{~m} * *$ & $20 \mathrm{mg} / \mathrm{kg}$ & $2.97 \pm 0.00$ & $2.03 \pm 0.01$ & $1.75 \pm 0.01$ & $1.53 \pm 0.02$ & $1.24 \pm 0.02$ & 33.22 & 42.43 & 49.50 & 59.07 \\
\hline 14 & $2 n *$ & $20 \mathrm{mg} / \mathrm{kg}$ & $2.99 \pm 0.01$ & $2.06 \pm 0.01$ & $1.90 \pm 0.01$ & $1.74 \pm 0.01$ & $1.58 \pm 0.01$ & 32.23 & 37.50 & 42.57 & 47.85 \\
\hline 15 & $20 * *$ & $20 \mathrm{mg} / \mathrm{kg}$ & $2.98 \pm 0.02$ & $1.79 \pm 0.01$ & $1.51 \pm 0.01$ & $1.25 \pm 0.01$ & $1.02 \pm 0.01$ & 41.11 & 50.32 & 58.74 & 66.36 \\
\hline 16 & $2 \mathbf{p}^{* *}$ & $20 \mathrm{mg} / \mathrm{kg}$ & $2.98 \pm 0.02$ & $1.78 \pm 0.01$ & $1.50 \pm 0.01$ & $1.22 \pm 0.01$ & $1.00 \pm 0.01$ & 41.44 & 50.65 & 59.73 & 66.99 \\
\hline 17 & $2 q^{* *}$ & $20 \mathrm{mg} / \mathrm{kg}$ & $2.98 \pm 0.02$ & $1.72 \pm 0.02$ & $1.48 \pm 0.01$ & $1.17 \pm 0.01$ & $0.97 \pm 0.01$ & 43.42 & 51.31 & 61.38 & 67.98 \\
\hline 18 & $2 \mathbf{r} * *$ & $20 \mathrm{mg} / \mathrm{kg}$ & $2.98 \pm 0.01$ & $1.75 \pm 0.01$ & $1.49 \pm 0.01$ & $1.20 \pm 0.01$ & $1.01 \pm 0.01$ & 42.43 & 50.98 & 60.39 & 66.66 \\
\hline 19 & $2 s * *$ & $20 \mathrm{mg} / \mathrm{kg}$ & $3.01 \pm 0.02$ & $2.03 \pm 0.01$ & $1.79 \pm 0.01$ & $1.58 \pm 0.01$ & $1.27 \pm 0.01$ & 33.22 & 41.11 & 47.85 & 58.08 \\
\hline 20 & $2 t * *$ & $20 \mathrm{mg} / \mathrm{kg}$ & $2.99 \pm 0.03$ & $2.11 \pm 0.02$ & $1.84 \pm 0.01$ & $1.63 \pm 0.01$ & $1.31 \pm 0.01$ & 30.59 & 39.47 & 46.20 & 56.76 \\
\hline 21 & $2 \mathbf{u}^{* *}$ & $20 \mathrm{mg} / \mathrm{kg}$ & $3.00 \pm 0.01$ & $2.15 \pm 0.01$ & $1.89 \pm 0.01$ & $1.69 \pm 0.01$ & $1.36 \pm 0.01$ & 29.27 & 37.82 & 44.22 & 55.26 \\
\hline 22 & $2 \mathrm{v} * *$ & $20 \mathrm{mg} / \mathrm{kg}$ & $3.01 \pm 0.02$ & $1.69 \pm 0.02$ & $1.28 \pm 0.03$ & $0.99 \pm 0.01$ & $0.57 \pm 0.01$ & 44.40 & 57.15 & 67.32 & 81.18 \\
\hline 23 & $2 \mathbf{w}^{* *}$ & $20 \mathrm{mg} / \mathrm{kg}$ & $3.02 \pm 0.01$ & $1.60 \pm 0.01$ & $1.17 \pm 0.02$ & $0.92 \pm 0.01$ & $0.55 \pm 0.01$ & 47.36 & 61.51 & 69.63 & 81.84 \\
\hline 24 & $2 x^{* *}$ & $20 \mathrm{mg} / \mathrm{kg}$ & $3.03 \pm 0.02$ & $1.50 \pm 0.02$ & $1.08 \pm 0.03$ & $0.85 \pm 0.03$ & $0.51 \pm 0.02$ & 50.65 & 64.44 & 71.94 & 83.16 \\
\hline 25 & Gum acacia $^{\mathrm{a}}$ & $2 \mathrm{~mL} / \mathrm{kg}$ & $3.00 \pm 0.01$ & $3.04 \pm 0.01$ & $3.04 \pm 0.01$ & $3.03 \pm 0.02$ & $3.03 \pm 0.01$ & - & - & - & - \\
\hline 26 & Pentazocine & $20 \mathrm{mg} / \mathrm{kg}$ & $1.71 \pm 0.01$ & $1.42 \pm 0.02$ & $1.00 \pm 0.03$ & $0.78 \pm 0.03$ & $0.46 \pm 0.02$ & 53.28 & 67.10 & 74.25 & 84.81 \\
\hline
\end{tabular}

a $2.0 \%(\mathrm{w} / \mathrm{v})$ of gum acacia was used as control

Data were analysed by one way ANOVA followed by Dunnet's test

$*$ significant, $P$ value $<0.05$; **highly significant, $P$ value $<0.001$

SEM: Standard error of means 
receiving $0.5 \%$ Tween solution. Group II was kept as standard, receiving indomethacin $(20 \mathrm{mg} / \mathrm{kg}$, p.o). Group III was kept as test, receiving synthesized compounds $(20 \mathrm{mg} / \mathrm{kg})$. After $30 \mathrm{~min}$, carrageenan solution $(0.1 \%$ in sterile $0.9 \% \mathrm{NaCl}$ solution $)$ in a volume of $0.1 \mathrm{~mL}$ was injected into the lateral malleolus of the sub-plantar region of the right hind paw of control as well as drug treated animals. The right hind paw volume was measured before and after carrageenan injection at various intervals $(30,60,90$ and $120 \mathrm{~min}$ ) using digital plethysmometer. The percentage antiinflammatory activity was calculated according to the following formula: \% Antiinflammatory activity = $(1-\mathrm{Vt} / \mathrm{Vc}) \times 100$, where $\mathrm{Vt}$ represents the mean in paw volume in rats tested with test compounds and $\mathrm{Vc}$ represents the mean increase in paw volume in control group of rats. Data are expressed as mean \pm SEM. The student $t$-test was applied to determine the significance of the difference between the control group and rats treated with the test compounds. The antiinflammatory activity of the 24 test compounds $\mathbf{2 a}-\mathbf{x}$ was compared with the standard drug indomethacin (table 4). At the same oral dose, indomethacin showed $77.91 \%$ inhibition of rat paw edema, whereas the tested com- pounds showed inhibition ranging from 9.69 to $73.93 \%$ after $120 \mathrm{~min}$. The results clearly indicate that among all the compounds, the indole derivatives $2 \mathbf{v}-\mathbf{x}$ potentially migrated the carrageenan-induced inflammation in rats and emerged as the most active compounds (69.69\%, 70.30\% and $73.93 \%$ inhibition, respectively). When the pyranone system with different substituents at C-3 position is fused with a piperanol ring $\mathbf{2} \mathbf{s}-\mathbf{u}$, moderate potency was observed and emerged as the second most active series. Replacement of piperanal moiety by a lipophilic naphthalene ring $\mathbf{2 0}-\mathbf{r}$ showed considerable decrease in activity. Finally, isocoumarins with different peripheral substituents but devoid of any heterocyclic core $\mathbf{2 a}-\mathbf{n}$ exhibited minimal antiinflammatory effect compared to other series. Among the peripheral substituents the nitro group $\mathbf{2 f}-\mathbf{h}$ was well-tolerated and offers enhanced activity over other substituents. A markedly reduced potency was observed when an alkyl chain is placed at the $\mathrm{C}-3$ position $\mathbf{2 d}, \mathbf{2 e}, \mathbf{2} \mathbf{I}$ and $\mathbf{2} \mathbf{m}$. It is our belief that molecular docking studies are required to study the hydrophobic interactions between the various atoms of the targeted compounds and aminoacid residues of the COX-II receptor, thus to reason out the observed activity.

Table 4. Antiinflammatory activity of synthesized compounds by carrageenan-induced paw edema method.

\begin{tabular}{|c|c|c|c|c|c|c|c|c|c|c|c|}
\hline \multirow[b]{2}{*}{ Entry } & \multirow[b]{2}{*}{ Treatments } & \multirow{2}{*}{$\begin{array}{l}\text { Dose } \\
\text { levels }\end{array}$} & \multicolumn{5}{|c|}{ Increase in paw volume (mean \pm SEM) } & \multicolumn{4}{|c|}{$\%$ Antiinflammatory activity } \\
\hline & & & $0 \mathrm{~min}$ & $30 \mathrm{~min}$ & $60 \mathrm{~min}$ & $90 \mathrm{~min}$ & $120 \mathrm{~min}$ & $30 \mathrm{~min}$ & $60 \mathrm{~min}$ & $90 \mathrm{~min}$ & $120 \mathrm{~min}$ \\
\hline 1 & $2 a^{* *}$ & $0 \mathrm{mg} / \mathrm{kg}$ & $1.66 \pm 0.01$ & $1.50 \pm 0.01$ & $1.46 \pm 0.02$ & $1.41 \pm 0.02$ & $1.35 \pm 0.01$ & 09.68 & 12.04 & 14.54 & 18.18 \\
\hline 2 & $2 b^{*}$ & $0 \mathrm{mg} / \mathrm{kg}$ & $1.66 \pm 0.02$ & $1.49 \pm 0.01$ & $1.42 \pm 0.01$ & $1.34 \pm 0.03$ & $1.29 \pm 0.01$ & 10.24 & 14.45 & 18.78 & 21.81 \\
\hline 3 & $2 c^{* *}$ & $20 \mathrm{mg} / \mathrm{kg}$ & $1.66 \pm 0.01$ & $1.52 \pm 0.01$ & $1.47 \pm 0.02$ & $1.42 \pm 0.02$ & $1.36 \pm 0.01$ & 08.40 & 11.44 & 13.93 & 17.57 \\
\hline 4 & $2 d^{* *}$ & $20 \mathrm{mg} / \mathrm{kg}$ & $1.66 \pm 0.02$ & $1.58 \pm 0.02$ & $1.53 \pm 0.01$ & $1.50 \pm 0.01$ & $1.47 \pm 0.01$ & 04.81 & 07.83 & 09.09 & 10.90 \\
\hline 5 & $2 \mathrm{e}^{* * *}$ & $20 \mathrm{mg} / \mathrm{kg}$ & $1.66 \pm 0.02$ & $1.59 \pm 0.02$ & $1.55 \pm 0.01$ & $1.52 \pm 0.01$ & $1.49 \pm 0.01$ & 04.21 & 06.62 & 07.87 & 09.69 \\
\hline 6 & $2 \mathbf{f}^{* *}$ & $20 \mathrm{mg} / \mathrm{kg}$ & $1.65 \pm 0.01$ & $1.35 \pm 0.02$ & $1.29 \pm 0.01$ & $1.25 \pm 0.01$ & $1.21 \pm 0.01$ & 18.78 & 22.28 & 24.24 & 26.66 \\
\hline 7 & $2 g *$ & $20 \mathrm{mg} / \mathrm{kg}$ & $1.66 \pm 0.02$ & $1.39 \pm 0.02$ & $1.33 \pm 0.01$ & $1.27 \pm 0.01$ & $1.24 \pm 0.01$ & 16.26 & 19.87 & 23.03 & 24.84 \\
\hline 8 & $2 h^{* *}$ & $20 \mathrm{mg} / \mathrm{kg}$ & $1.66 \pm 0.02$ & $1.36 \pm 0.02$ & $1.31 \pm 0.01$ & $1.26 \pm 0.01$ & $1.22 \pm 0.01$ & 18.07 & 21.08 & 23.63 & 26.06 \\
\hline 9 & $2 \mathrm{i}^{*}$ & $20 \mathrm{mg} / \mathrm{kg}$ & $1.66 \pm 0.01$ & $1.50 \pm 0.02$ & $1.47 \pm 0.01$ & $1.45 \pm 0.02$ & $1.37 \pm 0.02$ & 09.63 & 11.44 & 12.12 & 16.96 \\
\hline 10 & $2 \mathrm{j}^{* * *}$ & $20 \mathrm{mg} / \mathrm{kg}$ & $1.66 \pm 0.01$ & $1.56 \pm 0.02$ & $1.51 \pm 0.01$ & $1.48 \pm 0.01$ & $1.44 \pm 0.01$ & 06.02 & 09.03 & 10.30 & 12.72 \\
\hline 11 & $2 \mathbf{k}^{*}$ & $20 \mathrm{mg} / \mathrm{kg}$ & $1.66 \pm 0.01$ & $1.51 \pm 0.01$ & $1.48 \pm 0.02$ & $1.44 \pm 0.02$ & $1.36 \pm 0.02$ & 08.97 & 10.84 & 12.72 & 18.07 \\
\hline 12 & $21^{* *}$ & $20 \mathrm{mg} / \mathrm{kg}$ & $1.65 \pm 0.01$ & $1.55 \pm 0.02$ & $1.50 \pm 0.01$ & $1.47 \pm 0.01$ & $1.45 \pm 0.01$ & 06.62 & 09.09 & 10.90 & 12.12 \\
\hline 13 & $2 \mathbf{m}^{* *}$ & $20 \mathrm{mg} / \mathrm{kg}$ & $1.66 \pm 0.02$ & $1.54 \pm 0.02$ & $1.49 \pm 0.01$ & $1.46 \pm 0.01$ & $1.44 \pm 0.02$ & 07.22 & 10.24 & 11.44 & 12.72 \\
\hline 14 & $2 n *$ & $20 \mathrm{mg} / \mathrm{kg}$ & $1.66 \pm 0.01$ & $1.47 \pm 0.02$ & $1.40 \pm 0.01$ & $1.33 \pm 0.01$ & $1.28 \pm 0.01$ & 11.44 & 15.66 & 19.87 & 22.42 \\
\hline 15 & $20^{* *}$ & $20 \mathrm{mg} / \mathrm{kg}$ & $1.64 \pm 0.02$ & $1.34 \pm 0.02$ & $1.30 \pm 0.01$ & $1.24 \pm 0.01$ & $1.19 \pm 0$ & 19.27 & 21.68 & 24.84 & 27.87 \\
\hline 16 & $2 p^{* *}$ & $20 \mathrm{mg} / \mathrm{kg}$ & $1.66 \pm 0.01$ & $1.40 \pm 0.02$ & $1.37 \pm 0.01$ & $1.29 \pm 0.01$ & $1.25 \pm 0.02$ & 15.66 & 17.46 & 21.81 & 24.24 \\
\hline 17 & $2 q^{* *}$ & $20 \mathrm{mg} / \mathrm{kg}$ & $1.66 \pm 0.01$ & $1.37 \pm 0.02$ & $1.33 \pm 0.01$ & $1.27 \pm 0.01$ & $1.22 \pm 0.02$ & 17.46 & 19.69 & 23.03 & 26.06 \\
\hline 18 & $2 \mathbf{r}^{*}$ & $20 \mathrm{mg} / \mathrm{kg}$ & $1.66 \pm 0.01$ & $1.35 \pm 0.02$ & $1.31 \pm 0.01$ & $1.25 \pm 0.01$ & $1.20 \pm 0.02$ & 18.67 & 21.08 & 24.24 & 27.27 \\
\hline 19 & $2 s^{* * *}$ & $20 \mathrm{mg} / \mathrm{kg}$ & $1.65 \pm 0.02$ & $1.20 \pm 0.01$ & $1.01 \pm 0.01$ & $0.93 \pm 0.02$ & $0.73 \pm 0.01$ & 27.71 & 39.15 & 43.80 & 55.75 \\
\hline 20 & $2 t^{* *}$ & $20 \mathrm{mg} / \mathrm{kg}$ & $1.66 \pm 0.01$ & $1.25 \pm 0.01$ & $1.07 \pm 0.01$ & $1.00 \pm 0.02$ & $0.80 \pm 0.01$ & 24.69 & 35.54 & 39.39 & 51.51 \\
\hline 21 & $2 \mathbf{u}^{* *}$ & $20 \mathrm{mg} / \mathrm{kg}$ & $1.66 \pm 0.02$ & $1.24 \pm 0.01$ & $1.02 \pm 0.01$ & $0.98 \pm 0.02$ & $0.76 \pm 0.01$ & 25.30 & 38.55 & 40.60 & 53.93 \\
\hline 22 & $2 \mathrm{v}^{* *}$ & $20 \mathrm{mg} / \mathrm{kg}$ & $1.64 \pm 0.02$ & $0.95 \pm 0.01$ & $0.77 \pm 0.03$ & $0.64 \pm 0.03$ & $0.50 \pm 0.01$ & 42.77 & 53.61 & 61.21 & 69.69 \\
\hline 23 & $2 w^{* *}$ & $20 \mathrm{mg} / \mathrm{kg}$ & $1.65 \pm 0.02$ & $0.92 \pm 0.01$ & $0.75 \pm 0.01$ & $0.63 \pm 0.03$ & $0.49 \pm 0.01$ & 44.57 & 54.81 & 61.81 & 70.30 \\
\hline 24 & $2 x^{* *}$ & $20 \mathrm{mg} / \mathrm{kg}$ & $1.64 \pm 0.02$ & $0.99 \pm 0.01$ & $0.79 \pm 0.01$ & $0.68 \pm 0.03$ & $0.43 \pm 0.01$ & 40.36 & 52.40 & 58.78 & 73.93 \\
\hline 25 & Tweer & $2 \mathrm{~mL} / \mathrm{kg}$ & $1.67 \pm 0.01$ & $1.66 \pm 0.01$ & $1.66 \pm 0.01$ & $1.65 \pm 0.02$ & $1.65 \pm 0.02$ & - & - & - & - \\
\hline 26 & Indomethacin & $20 \mathrm{mg} / \mathrm{kg}$ & $1.58 \pm 0.01$ & $1.19 \pm 0.03$ & $0.91 \pm 0.04$ & $0.55 \pm 0.03$ & $0.36 \pm 0.02$ & 28.31 & 45.18 & 66.6 & 77.91 \\
\hline
\end{tabular}

${ }^{\mathrm{a}} 0.5 \%(\mathrm{w} / \mathrm{v})$ of Tween 80 was used as control

Data were analysed by one way ANOVA followed by Dunnet's test

$*$ significant, $P$ value $<0.05$; **highly significant, $P$ value $<0.001$

SEM: Standard error of means 


\section{Conclusion}

We have reported here a brief investigation on the sequential hydrolysis/cycloisomerization of $O$ (alkynyl)benzamides with triflic acid under microwave condition. This methodology has allowed us to construct substituted isocoumarins in a regioselective manner. To the best of our knowledge, these are the first examples of direct synthesis of isocoumarins from $o$ (alkynyl)benzamides under super acid catalysis. This chemistry significantly broadens the synthetic utility of $o$-(alkynyl)benzamides and highlights the ability of triflic acid to operate two dissimilar reactions in the same reaction flask. The biological potential of the synthesized compounds were evaluated for their antinociceptive and antiinflammatory activities using in vivo rodent models, which indicate a comparable activity against the reference drugs. The biological evaluation led to the finding that pyranone fused with indole rings exhibited excellent antinociceptive as well as antiinflammatory activities. Studies addressed toward the application of this methodology to natural products and identification of reaction intermediates using deuterium labelling experiments are under investigation.

\section{Acknowledgements}

The authors are thankful to the Council of Scientific and Industrial Research (CSIR), New Delhi, for the financial support. CP acknowledges Mr. D Muralidharan, Scientist-G, Organic Chemistry Division, Central Leather Research Institute (CLRI), Chennai, for his valuable suggestions.

\section{References}

1. Howells R D and McCown J D 1977 Chem. Rev. 7769

2. Stang P J and White M R 1983 Aldrichimica Acta 1615

3. (a) Puglici A, Lee A-L, Schrock R R and Hoveyda A H 2006 Org. Lett. 8 1871; (b) Bennasar M-L, Zulaica E and Tummers S 2004 Tetrahedron Lett. $\mathbf{4 5} 6283$

4. Haszeldine R N and Kidd J M 1954 J. Chem. Soc. 4228

5. Gramstad T and Haszeldine R N 1956 J. Chem. Soc. 173

6. (a) Dumeunier R and Markó I E 2004 Tetrahedron Lett. 45 825; (b) Loh T-P, Hu Q-Y and Ma L-T 2002 Org. Lett. 4 2389; (c) Olah G A, Wu A-h 1991 Synthesis 407; (d) Rosenfeld D C, Shekhar S, Takemiya A, Utsunomiya M and Hartwig J F 2006 Org. Lett. 8 4179; (e) Villemin D, Bar N and Hammadi M 1997 Tetrahedron Lett. 38 4777; (f) Abid M, Teixeira L and Török B 2007 Tetrahedron Lett. 48 4047; (g) Li Z, Zhang J, Brouwer C, Yang C-G, Reich N-W and He C 2006 Org. Lett. 8 4175; (h) Elford T G, Arimura Y, Yu S H and Hall D G 2007 J. Org. Chem. 72 1276; (i) Abid M, Teixeira L and Török B 2008 Org. Lett. 10 933; (j) Corey E J, Shibata T and
Lee T W 2002 J. Am. Chem. Soc. 124 3808; (k) Koltunov K Y 2007 Tetrahedron Lett. 48 5631; (1) Reddy B V S, Ramesh K, Ganesh A V, Kumar G G K S N, Yadav J S and Grée R 2011 Tetrahedron Lett. 52 495; (m) Jin T, Uchiyama J, Himuro M and Yamamoto Y 2011 Tetrahedron Lett. 52 2069; (n) Safina L Y, Selivanova G A, Koltunov K Y and Shteingarts V D 2009 Tetrahedron Lett. 50 5245; (o) Li A, DeSchepper D J and Klumpp D A 2009 Tetrahedron Lett. 50 1924; (p) Bodnar B S and Miller M J 2009 Tetrahedron Lett. 50 796; (q) Mendoza O, Rossey G and Ghosez L 2011 Tetrahedron Lett. 52 2235; (r) Klumpp D A, Zhang Y, Kindelin P J and Lau S 2006 Tetrahedron 62 5915; (s) Klumpp D A, Yeung K Y, Prakash G K S and Olah G A 1998 J. Org. Chem. 63 4481; (t) Yamamoto Y, Gridnev I D, Patil N T and Jin T 2009 Chem. Commun. 5075

7. Ramsden C A, Scriven E F V and Taylor R J K 2008 Comprehensive heterocyclic chemistry, III edition; New York: Elsevier

8. (a) Bunin B A 1998 The combinatorial index; London: Academic Press; (b) Obrecht D and Villalgordo J M 1998 Solid-supported combinatorial and parallel synthesis of small-molecular-weight compound libraries; Elsevier; (c) In: Combinatorial chemistry: Synthesis, analysis, screening; Jung G, Ed.; Weinheim: WileyVCH 1999; (d) Combinatorial chemistry and molecular diversity in drug discovery; E M Gordon and J F Kerwin Jr (eds); New York: John Wiley \& Sons 1998

9. Uchiyama M, Ozawa H, Takuma K, Matsumoto Y, Yonehara M, Hiroya K and Sakamoto T 2006 Org. Lett. 85517

10. Bianchi G, Chirani M, Marinelli F, Rossi L and Arcadi A 2010 Adv. Synth. Catal. 352136

11. Roy S, Roy S, Neuenswander B, Hill D and Larock R C 2009 J. Comb. Chem. 111128

12. (a) Praveen C, Dheenkumar P, Muralidharan D and Perumal P T 2010 Bioorg. Med. Chem. Lett. 20 7292; (b) Praveen C, Kumar K H, Muralidharan D and Perumal P T 2008 Tetrahedron 64 2369; (c) Praveen C, Parthasarathy K and Perumal P T 2010 Synlett. 1635; (d) Praveen C, Jegatheesan S and Perumal P T 2009 Synlett 2795; (e) Praveen C, Kalyanasundaram A and Perumal P T 2010 Synlett 777; (f) Praveen C, Sagayaraj Y W and Perumal P T 2009 Tetrahedron Lett. 50, 644; (g) Praveen C, Kiruthiga P and Perumal P T 2009 Synlett. 1990; (h) Praveen C, Karthikeyan K and Perumal P T 2009 Tetrahedron 65 9244; (i) Praveen C and Perumal P T 2011 Synlett 521; (j) Praveen C, Iyyappan C and Perumal P T 2010 Tetrahedron Lett. 51 4767; (k) Praveen C, Ayyanar A and Perumal P T 2011 Bioorg. Med. Chem. Lett. 21 4072; (1) Praveen C, Ayyanar A and Perumal P T 2011 Bioorg. Med. Chem. Lett. 21 4170; (m) Balakrishnan B, Praveen C, Seshadri P R and Perumal P T 2011 Acta Crystallogr. E67 o1575; (n) Praveen C, Iyyappan C, Girija K, Kumar K S and Perumal P T 2012 J. Chem. Sci. 124 451; (o) Praveen C, Iyyappan C, Perumal P T and Girija K 2012 Indian J. Chem. Soc. 51B 498; (p) Praveen C, Nandakumar A, Dheenkumar P, Muralidharan D and Perumal P T 2012 J. Chem. Sci. 124609

13. For the cyclization of 2-(alkynyl)benzamides to isoindolinones and isoquinolinones, see: (a) Yao $\mathrm{T}$ and Larock R C 2003 J. Org. Chem. 68 5936; (b) Koseki 
Y, Kusano S, Sakata H and Nagasaka T 1999 Tetrahedron Lett. 40 2169; (c) Kundu N G, Khan M W and Mukhopadgyay R 1999 Tetrahedron 55 12361; (d) Kundu N G, Khan M W 2000 Tetrahedron 564777

14. For the cyclization of 2-(alkynyl)benzoic acids to isocoumarins and phthalides, see: (a) Mehta S Waldo J P and Larock R C 2009 J. Org. Chem. 74 1141; (b) Yao T and Larock R C 2003 J. Org. Chem. 68 5936; (c) Peuchmaur M, Lisowski V, Gandreuil C, Maillard L T, Martinez J and Hernandez J-F 2009 J. Org. Chem. 74 4158; (d) Biagetti M, Bellina F, Carpita A, Stabilea P and Rossi R 2002 Tetrahedron 58 5023; (e) Roy S, Roy S, Neuenswander B, Hill D and Larock R C 2009 J. Comb. Chem. 11 1128; (f) Sakamoto T, An-Naka M, Kondo Y and Yamanaka H 1986 Chem. Pharm. Bull. 34 2754; (g) Subramanian V, Batchu V R, Barange D and Pal M 2005 J. Org. Chem. 70 4778; (h) Uchiyama M, Ozawa H, Takuma K, Matsumoto Y, Yonehara M, Hiroya K and Sakamoto T 2006 Org. Lett. 8 5517; (i) Raju S, Batchu V R, Swamy N K, Dev R V, Sreekanth B R, Babu J M, Vyas K, Kumar P R, Mukkanti K, Annamalai P and Pal M 2006 Tetrahedron 62 9554; (j) Sashida H, Kawamukai A 1999 Synthesis 1145; (k) Liao H-Y and Cheng C-H 1995 J. Org. Chem. 60 3711; (1) Liang Y, Xie Y-X and Li J-H 2007 Synthesis 400; (m) Bras G L, Hamze A, Messaoudi S, Provot O, Calvez P-B L, Brion J-D and Alami M 2008 Synthesis 1607

15. For reviews highlighting the significance of microwave in organic transformations, see: (a) Caddick S 1995 Tetrahedron 51 10403; (b) Lidström P, Tierney J, Wathney B and Westman J 2001 Tetrahedron 57 9225; (c) Wathey B, Tierney J, Lidstrom P and Westman 2002 J. Drug Discovery Today 7 373; (d) Alcázar J, Diels G and Schoentjes B 2007 Comb. Chem. High Throughput Screening 10 918; (e) Caddick S and Fitzmaurice R 2009 Tetrahedron 65 3325; (f) De la Hoz A, Díaz-Ortiz A and Moreno A 2005 Chem. Soc. Rev. 34 164; (g) Galema S A 1997 Chem. Soc. Rev. 26 233; (h) Rajak H and Mishra P 2004 J. Sci. Ind. Res. 63641

16. (a) Goldstein H and Grampoloff A V 1930 Helvetica Chimica Acta 13 310; (b) Kenner J and Turner H A 1928 J. Chem. Soc. 2340; (c) Stanley W M, McMahon E and Adams R 1993 J. Am. Chem. Soc. 55 706; (d) Harayama T and Shibaike K 1998 Heterocycles 49191

17. (a) Wu M-J, Chang L-J, Wei L-M and Lin C-F 1999 Tetrahedron 55 13193; (b) Couture A, Grandclaudon P 1986 Synthesis 576

18. Yao T and Larock R C 2005 J. Org. Chem. 701432

19. Kundu N G and Khan M W 2000 Tetrahedron 564777

20. Travis B R, Sivakumar M, Hollist G O and Borhan B 2003 Org. Lett. 51031

21. Doyle F P, Ferrier W, Holland D O, Mehta M D and Nayler J H C 1956 J. Chem. Soc. 2853

22. (a) Chashi T, Sada T, Fujimoto H, Nagayama C, Sugino E and Hibino S 1997 J. Org. Chem. 62 2535; (b) Sakamoto T, Nagano T, Kondo Y and Yamanaka H 1988 Chem. Pharm. Bull. 362248

23. (a) Dehaen W and Hassner A 1991 J. Org. Chem. 56 896; (b) Tsotinis A, Afroudakis P A, Davidson K, Prashar A and Sugden D 2007 J. Med. Chem. 50 6436; (c) Li C-F, Liu H, Liao J, Cao Y-J, Liu X-P and Xiao
W-J 2007 Org. Lett. 9 1847; (d) Sechi M, Derudas M, Dallocchio R, Dessí A, Bacchi A, Sannia L, Carta F, Palomba M, Ragab O, Chan C, Shoemaker R, Sei S, Dayam R and Neamati N 2004 J. Med. Chem. 47 5298; (e) Forbes I T, Kennet G A, Gadre A, Ham P, Hayward C J, Martin R T, Thompson M, Wood M D, Baxter G S, Glen A, Murphy O E, Stewart B A and Blackburn T P 1993 J. Med. Chem. 38 1104; (f) Kikugawa Y and Miyake Y A 1981 Synthesis 461; (g) Bergman J and Sand P 1993 Tetrahedron 436085

24. Larock R C, Varaprath S, Lau H H, Fellows C A 1984 J. Am. Chem. Soc. 1065274

25. Marchal E, Uriac P, Legouin B, Toupet L and van de Weghe P 2007 Tetrahedron 639979

26. Chin L-Y, Lee C-Y, Lo Y-H and Wu M-J 2008 J. Chin. Chem. Soc. 55643

27. (a) Batu G and Stevenson R 1980 J. Org. Chem. 45 1532; (b) Liao H-Y and Cheng C-H 1995 J. Org. Chem. 60 3711

28. Ohta S, Kamata Y, Inagaki T, Masuda Y, Yamamoto S, Yamashita M and Kawasaki I 1993 Chem. Pharm. Bull. 411188

29. Woon E C Y, Dhami A, Mahon M F and Threadgill M D 2006 Tetrahedron 624829

30. Elix J A and Murphy D P 1975 Aust. J. Chem. 281559

31. Rossi R, Carpita A, Bellina F, Stabilea P and Mannina L 2003 Tetrahedron 592067

32. Lewis C N, Spargo P L and Staunton J 1986 Synthesis 944

33. Cherry K, Parraim J-L, Thibonnet J, Duchêne A and Abarbri M 2005 J. Org. Chem. 706669

34. Hauser F M, Dorsch W A and Mal D 2002 Org. Lett. 4 2237

35. Miura M, Tsuda T, Satoh, Pivsa-Art S and Nomura M 1998 J. Org. Chem. 635211

36. (a) Ghosh M N 2005 Fundamentals of experimental pharmacology, 3rd Edition, Kolkatta: Hilton and Co. 190-7; (b) Kale S R and Kale R R 1994 Practical pharmacology and toxicology, 1st Edition, Pune: Nirali Prakashan, 56

37. OECD 1987 Guidelines for testing chemicals, No. 401, Acute Oral Toxicity (Paris), 1

38. (a) Sonogashira K, Tohda Y and Hagihara N 1975 Tetrahedron Lett. 16 4467; (b) Tykwinsky R R 2003 Angew. Chem. 115 1604; (c) Tykwinsky R R 2003 Angew. Chem. Int. Ed. 421566

39. It was expected that the evolution of toxic fumes of triflic acid could be somewhat controlled by using toluene which forms the top layer. For related discussion, see: Rombouts F, Franken D, Martínez-Lamenca C, Braeken M, Zavattaro C, Chen J and Trabanco A A 2010 Tetrahedron Lett. $\mathbf{5 1} 4815$

40. No reaction was observed at room temperature

41. The physical and spectral data of the product matched with the literature value, see: ref. 23

42. Hydrolysis of benzamide to benzoic acid in excellent yield was achieved under similar conditions

43. For the cyclization of 2-(alkynyl)benzoic acids to both phthalides and isocoumarins, see: (a) Sakamoto T, An-naka M, Kondo Y and Yamanaka H 1986 Chem. Pharm. Bull. 34 2754; (b) Sashida H and Kawamukai A 1999 Synthesis 1145; (c) Inack-Ngi S, Rahmani R, 
Commeiras L, Chouraqui G, Thibonnet J, Duchêne A, Abarbri M and Parrain J-L 2009 Adv. Synth. Catal. 351 779; (d) Kundu N G and Pal M J 1993 Chem. Soc. Chem. Commun. 86. Also see: ref. 24 and 30.

44. For discussion, see: (a) Li X and Liao S $2009 \mathrm{~J}$. Mol. Struct. (THEOCHEM) 897 66; (b) Olha G A; Batamack P, Deffieux D; Török B, Wang Q, Molnár A and Praksah G K S 1996 Appl. Catal. A-Gen. 146107

45. (a) Trani A, Dallanoce C, Panzone G, Ripamonti F, Goldstein B P and Ciabatti R 1997 J. Med. Chem. 40 967; (b) Lee J H, Park Y J, Kim H S, Hong Y S, Kim K W and Lee J J 2001 J. Antibiot. 54 463; (c) Matsuda H, Shimoda H, Yamahara J and Yoshikawa M 1998 Bioorg. Med. Chem. Lett. 8215

46. (a) Bauta W E, Lovett D P, Cantrell Jr W R and Burke B D 2003 J. Org. Chem. 68 5967; (b) Whyte A C, Gloer J B, Scott J A and Mallock D 1996 J. Nat. Prod. 59 765; (c) Furuta T, Fukuyama Y and Asakawa Y 1986 Phytochemistry 25 517; (d) Yoshikawa M, Uchida E, Chatani N, Murakami N and Yamahara J 1992 J. Chem. Pharm. Bull. 40 3121; (e) Yoshikawa M, Uchida E, Naitoh Y, Inoue K, Matsuda H, Shimoda H, Yamahara J and Murakami N 1994 J. Chem. Pharm. Bull. 42 2225; (f) Yoshikawa M, Matsuda H, Shimoda H, Shimada H, Harada E, Naitoh Y, Miki A, Yamahara J and Murakami N 1996 J. Chem. Pharm. Bull. 44 1440; (g) Bürki N, Michel A and Tabacchi R 2003 Phytopathol. Mediterr. 42 191; (h) Xin Z-H, Li-Tian, Zhu T-J, Wang W-L, Du L, Fang Y-C, Gu Q-Q and Zhu W-M 2007 Arch. Pharm. Res. $\mathbf{3 0} 816$

47. (a) Abramovitch R A and Cue B W Jr 1973 Heterocycles 1 227; (b) Pletnev A A, Tian Q and Larock R C 2002 J. Org. Chem. 679276

48. For discussion, see: Kundu N G, Pal M and Nandi B 1998 J. Chem. Soc. Perkin Trans. 1561

49. (a) Winter C A, Risley E A and Nuss G W 1963 J. Pharmacol. Exp. Ther. 141 369; (b) Amir M, Kumar H and Khan S A 2008 Bioorg. Med. Chem. Lett. 18 918; (c) Winter C A, Risley E A and Nuss G W 1962 Proc. Soc. Exp. Biol. Med. 111544

50. Seibert K, Zhang Y, Leahy K, Hauser S, Masferrer J, Perkins W, Lee L and Isakson P 1994 Proc. Natl. Acad. Sci. USA 9112013

51. Vogel H G 2002 Drug discovery and evaluationPharmacological assays, 2nd Edition, New York: Springer, 697 